\title{
EFECTOS DE FUERZAS DINÁMICAS APLICADAS POR EQUIPOS DE CONSTRUCCIÓN SOBRE SUELOS BLANDOS DE LA SABANA DE BOGOTÁ
}

\section{EFFECTS OF DYNAMIC FORCES APPLIED BY CONSTRUCTION EQUIPMENT ON SOFT SOILS OF "SABANA DE BOGOTÁ"}

\author{
Castillo V, Juan. * Diaz D, Nicolas. * MSc. Torres R, Carlos. * \\ $\frac{\frac{\text { juancastilloveusantotomas.edu.co }}{\text { nicolasdiazdeusantotomas.edu.co }}}{\text { carlos.torresreusantotoms.edu.co }}$
}

*Facultad de Ingeniería Civil, Universidad Santo Tomás, Bogotá D.C., Colombia,

\section{RESUMEN}

La dinámica de suelos como ciencia física que hace parte de la ingeniería geotécnica estudia los problemas derivados de las fuerzas vibratorias dinámicas. En la presente investigación se analizan dichas fuerzas mediante la transmisión de ondas a través del suelo como un medio poroso, enfocándose en aquellas producidas por la maquinaria y equipos utilizados en procesos constructivos propios de la ingeniería civil, las cuales pueden conducir a una afectación de las propiedades del suelo y un posible colapso de este producido por la similitud de contenido frecuencial entre ambos elementos. El presente artículo está basado en la simulación de condiciones naturales del suelo, mediante la realización de ensayos de columna resonante, sometiendo un espécimen de suelo a un estado de vibración forzada a través de fuerzas torsionales, variando la frecuencia de excitación para lograr conocer la frecuencia de resonancia de la muestra en estudio, con el ánimo de realizar un comparativo de la misma respecto a la frecuencia promedio máxima de vibración de máquinas y equipos comúnmente utilizados en construcción. Para el desarrollo de esta investigación se realizó la selección de la zona de estudio, la cual se encuentra ubicada en el municipio de Tenjo, Cundinamarca, Colombia, donde se llevaron a cabo dos perforaciones con una profundidad máxima de 6 metros cada una, mediante el uso de SPT y muestreo mediante tubería de pared delgada "Shelby. La zona de estudio corresponde según el Servicio Geológico Colombiano en su caracterización geológica al tipo Q1-I, clasificado como un depósito del eón fanerozoico de la era cenozoica en el periodo cuaternario, dicho depósito se encuentra compuesto por arcillas blandas y turbas, las cuales poseen contenidos de arena y niveles delgados de gravas, que son localmente capas de diatomitas. Para la caracterización sísmica de dicha zona es tenida en cuenta la gráfica zonal de amenaza sísmica presente en la NSR-10 título A, donde es posible apreciar que el lugar de estudio se encuentra en una zona de amenaza sísmica intermedia. Teniendo en cuenta que el objeto de análisis de esta investigación es un tipo de suelo presente en la sabana de Bogotá, caracterizado como arcilla blanda de alta plasticidad (CH) que contiene minerales como illita, kaolinita y montmorillonita. Se realizó un estudio con base en la respuesta del suelo frente a cargas dinámicas impuestas, con lo cual se determinaron algunos patrones de comportamiento típico que éste presenta, esto con el fin de generar interés y consciencia dentro del gremio profesional de la ingeniería civil, conduciendo así a la prevención de posibles imprevistos que ocasionen consecuencias negativas en futuras construcciones.

Palabras claves: Contenido frecuencial, cargas dinámicas, simulación, maquinaria y equipo, columna resonante, depósitos de arcilla.

ABSTRACT---The soil dynamics as a physic science of geotechnical engineering that studies problems arising from dynamic vibratory forces. This investigation analyzes these forces through the wave transmission through the soil as porous medium, focusing on waves produced by machinery and equipment used in civil engineering construction process that could conduce to a soil properties affectation and a possible collapse of them produced by frequency content similarity by both elements. This article is based on the study of dynamic properties of natural soil through resonant column tests, submitting a specimen of soil to forced vibratory status through torsional forces, varying the excitation frequency to know resonance frequency and compares to maximum frequency vibration of machines and equipment commonly used in construction. For development of this investigation it chooses a study zone located in Tenjo, Cundinamarca, Colombia, where two perforations were made with maximum depth of $6 \mathrm{~m}$ through SPT and thin wall shelby tube sampler. According to Colombian geological service the study zone corresponds in them characterization to Q1-I type, classified as a deposit of the Phanerozoic aeon of the Cenozoic era in the Quaternary period composed by soft clays and organic soil with sand content and thin levels of gravel which are locally layers of diatomites. To seismic characterization of study zone is having in mind seismic threat graphic present in title A of NSR-10, where is possible to appreciate that study point is located in an intermediate seismic threat zone. Considering that the analysis object of this investigation is the study of a soil type present in the "Sabana de Bogota'" characterized as soft fat clay with high plasticity (CH) and mineral content as illite, kaolinite and montmorillonite. A study was made based on the response of the soil to imposed dynamic loads, which determined some patterns of typical behavior that it presents, this in order to generate interest and awareness within the professional association of civil engineering, thus leading to the prevention of possible unforeseen that cause negative consequences in future constructions.

Key words: frequency content, dynamic loads, simulation, machinery and equipment, resonant column, clay deposits. 


\section{Introducción}

Existen fuerzas de la naturaleza que son poco estudiadas y que pueden afectar las obras de ingeniería, dadas las características particulares de los suelos que las soportan, como lo son las fuerzas dinámicas. Aunque existe diversidad entre estas, la presente investigación está centrada en analizar los efectos de fuerzas producidas por la vibración de maquinaria pesada usada en la construcción de obras civiles, debido a esto, se hace necesario profundizar desde la investigación en temas de estudio que reproduzcan beneficios en la práctica de la ingeniería civil.

El presente artículo contiene los resultados del estudio del comportamiento dinámico de un suelo blando de la sabana de Bogotá, y la influencia de sus propiedades en el comportamiento de estructuras vibratorias usadas en la construcción; para ello se cuenta con información histórica y antecedentes los cuales son la el marco de referencia para este artículo, y la guía para los análisis correspondientes, los cuales dan inicio con la selección específica de un sector de estudio debidamente georreferenciado, del cual se extrajeron muestras de suelo para su posterior caracterización y clasificación, logrando así determinar sus propiedades mecánicas, además se llevaron a cabo ensayos de columna resonante los cuales permitieron determinar propiedades fundamentales para el entendimiento del comportamiento dinámico de los suelos.

\section{Estado del arte}

La dinámica de suelos ha evolucionado como una disciplina independiente, desarrollando procedimientos tanto teóricos como experimentales. En países como Alemania, EE. UU., la antigua URSS se conocían antecedentes de efectos como grandes asentamientos diferenciales y vuelcos de estructuras como producto de fuerzas dinámicas. La dinámica de suelos se puede aplicar a la solución de cimentaciones para maquinaria, respuestas sísmicas, explosiones, compactación, licuación, transito, y condiciones locales. Parte de la caracterización de los problemas dinámicos de suelos, es que, si las cargas aplicadas a una masa de suelo inducen fuerzas de inercia de magnitud comparable a las fuerzas estáticas, se requiere la determinación de los parámetros del suelo y el desarrollo de herramientas de cálculo que tomen en cuenta esta condición. Estudia distintas temáticas tales como: comportamiento y respuesta del suelo durante la aplicación de carga, principales propiedades dinámicas (módulo de corte, relación de amortiguamiento, deformaciones), comportamiento de ondas, principalmente, la respuesta dinámica del suelo está dada en función de su capacidad de disipar energía a través de deformación partiendo de la curva esfuerzo cortante vs deformación angular, la cual arroja el módulo de corte G [1].

La dinámica de suelos al evaluar y analizar los campos frecuentes de Ingeniería Sísmica relaciona las propiedades dinámicas del suelo con procesos y fenómenos indispensables como lo son las ondas, que mide y valora especialmente su velocidad de corte. Esta ciencia requiere de un profundo análisis para lograr comprender la incidencia que su estudio puede generar en la actualidad; vibraciones a causa de maquinaria o el paso de vehículos, sismos y la ejecución de fundaciones generan modificaciones en el estado actual del terreno, El estudio de la dinámica de suelos necesita del empleo de diferentes herramientas de orden conceptual, numérico y experimental, siendo así la contraprestación al campo investigativo de gran utilidad ya que mediante esto se logra entender el comportamiento del suelo ante cargas dinámicas [2].
El ensayo de Columna Resonante es un método de laboratorio para conocer las propiedades dinámicas de los depósitos de suelo, el cual cubre la determinación del módulo de corte, las características de amortiguamiento, la velocidad de onda de corte y el módulo de Young, para muestras cilíndricas de suelo en condiciones alteradas $\mathrm{o}$ inalteradas. Por otro parte, se tiene en [3, 4], dos importantes trabajos de investigación donde implementan el ensayo de columna resonante para estudiar cómo se comporta el suelo. Este ensayo permite medir las propiedades dinámicas del suelo a pequeñas deformaciones (módulo cortante y relación de amortiguamiento), el equipo implementado en el laboratorio de suelos de la Universidad permite ejecutar otros ensayos los cuales tienen la misma finalidad, estos son: Bender Elements y torsión cíclica, en el laboratorio se pueden hallar respuestas a bajas deformaciones, altas deformaciones y velocidades de onda, a través de ensayos de columna resonante, triaxial cíclico y Bender Elements respectivamente.

El objetivo de los ensayos dinámicos es identificar las propiedades dinámicas del suelo como el módulo equivalente de Young (Eeq), el módulo de esfuerzo cortante (G), el módulo de deformación

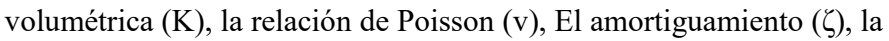

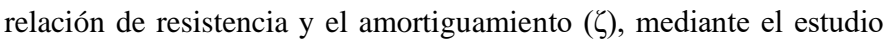
del comportamiento de la longitud de onda, la amplitud y el periodo resultantes de la transmisión de ondas a través del material en estudio, que para el caso de los suelos pueden ser ondas de cuerpo u ondas de superficie; Las cuales son esenciales para analizar la respuesta sísmica y la estabilidad bajo condiciones de carga [5].

El comportamiento elástico se da a pequeñas deformaciones que están en el orden de $1 \times 10^{-4} \%$, mientras que la falla del material generalmente se alcanza con deformaciones que oscilan entre $1 \times 10^{-4}$ $\%$ y $1 \times 10^{-2} \%$, debido a esto se llevan a cabo ensayos de columna resonante para evaluar el comportamiento a la falla. Además, cabe resaltar que la rigidez de los materiales se puede determinar mediante la deformación de estos teniendo en cuenta la distorsión angular sobre sus ejes X y Y.

Las frecuencias encontradas en suelos arcillosos ante eventos de carga dinámicas como fuerzas de sismos, explosiones o las producidas por maquinaria de construcción, producen un comportamiento atípico en el suelo, por lo que analizar este tipo de fenómenos con lleva un arduo análisis. Para llevar a cabo esta tarea es importante conocer las propiedades índices y mecánicas del suelo en estudio, ya que con esto se puede someter y evaluar el suelo a cargas estáticas y cíclicas. De acuerdo con Kitovas [6], se demostró que las propiedades mecánicas de la arcilla cambiaron completamente, por lo que la resistencia de ese suelo vario en gran proporción, esto sometiendo la muestra de suelo a una frecuencia de 10 a $50 \mathrm{~Hz}$. Los autores de este artículo sugieren que el comportamiento de la arcilla bajo la influencia de vibraciones se asimila a una arcilla no drenada. 


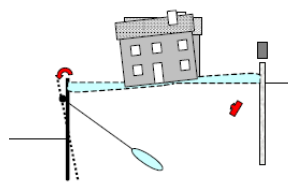

I. Differential settlements or heave due to static soil movements

III. Settlement and/or strength loss due to cyclic effects

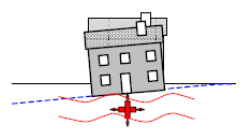

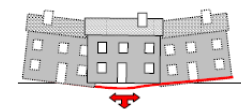

II. Damage in structure due to ground distortion

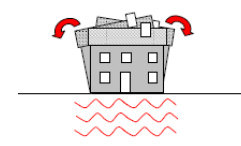

V. Structural damage due to dynamic effects
Fig. 1 Diferentes tipos de daños en edificios observados en relación con las actividades de construcción, por Massarsch [7].

Acorde con Massarsch [7], en su artículo de "asentamientos y daños causados por las vibraciones inducidas por la construcción”, se sabe que las actividades realizadas en ingeniería civil, para ser más específico en el área de construcción pueden llegar a generar vibraciones y de esta formar afectar de manera considerable a las estructuras cercanas a la zona, asimismo esta afectación se puede presentar de diferentes formas tal como se evidencia en la Figura 1. Generalmente los daños ocurren como resultado de la distorsión entre el soporte terrestre y asentamientos diferenciales, cabe resaltar que las vibraciones que se generan en suelos no cohesivos pueden dar origen a asentamientos debido a cargas cíclicas. De acuerdo con distintos ensayos dinámicos y cíclicos de laboratorio se encontró que la rigidez y la fuerza del suelo disminuye cuando se excede el máximo nivel de tensión critica. Por otro lado, el autor hace referencia en que la magnitud de los asentamientos o daños dependen principalmente de la tensión de cizallamiento y el número de ciclos de vibración.

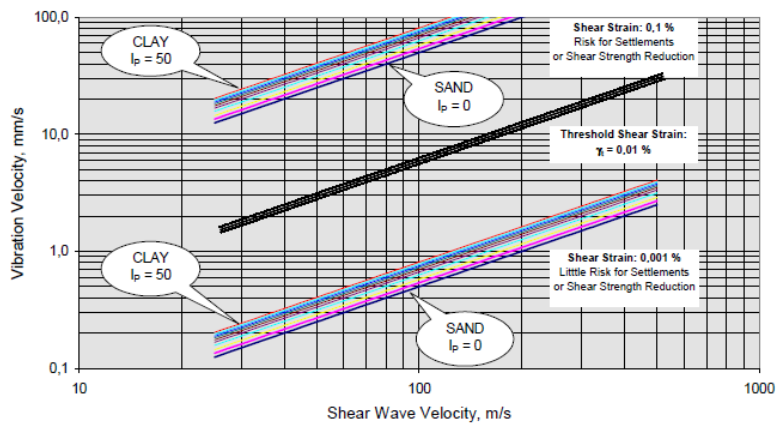

Fig. 2 Estimación del riesgo de asentamientos o reducción de la fuerza por la velocidad de la vibración como función de la velocidad de la onda de cizallamiento para diferentes niveles de tensión de cizallamiento, por Massarsch [7].

Tal como se puede observar en la Figura 2 se ha establecido una relación entre los esfuerzos verticales, de corte y la velocidad de vibración del suelo para arcillas y arenas por lo que con estos datos se pueden estimar en que valores se genera el máximo nivel de esfuerzo y por ende cuando empieza a verse la perdida de rigidez y fuerza en el suelo [7].

Por otra parte, en el documento "El impacto de la vibración producida por la construcción en las estructuras adyacentes" realizado por la empresa JS Held [8], hablan sobre como la máxima velocidad pico de las partículas permitidas para la construcción cambia con la frecuencia como se evidencia en la Figura 3, además el autor plantea que para realizar un análisis de las vibraciones generadas por la construcción en una estructura, se debe evaluar si la afectación es directa o indirecta, siendo esta primera causada por las ondas superficiales que afectan los cimientos de la estructura y hacen que estos trasmitan la energía hacia el resto de la estructura que soportan, por lo que provocan esfuerzos de flexión en muros y otros elementos estructurales, esto debido a que la energía de onda es absorbida y reflejada a través de los materiales de la estructura, en cuanto al efecto indirecto es cuando las ondas producidas por la vibración afectan directamente al suelo sobre el cual esta soportada la estructura, produciendo asentamientos diferenciales y posibles colapsos de esta.

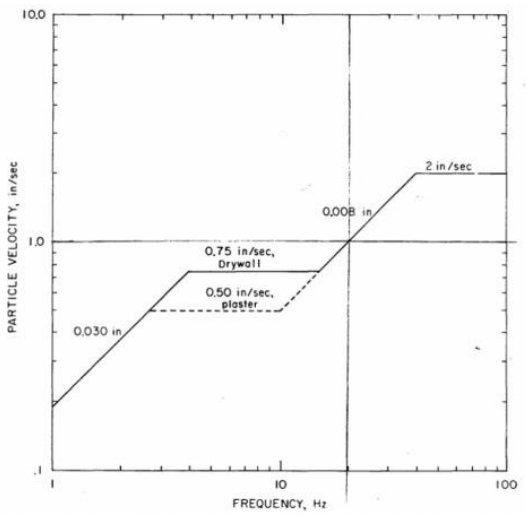

Fig. 3 Criterios de la Oficina de Minas de los Estados Unidos para el impacto directo, por JS Held [8].

Según el autor Hal Amick en "Construction vibrations and their impact on vibration-sensitive facilities" [9], se tiene una serie de curvas de las típicas vibraciones del suelo debido a la construcción como se puede observar en la Figura 4, donde se muestra la velocidad pico de la partícula y la distancia desde la fuente. Con esta Figura se puede observar el rango hasta donde pueden llegar las ondas generadas por equipos de construcción.
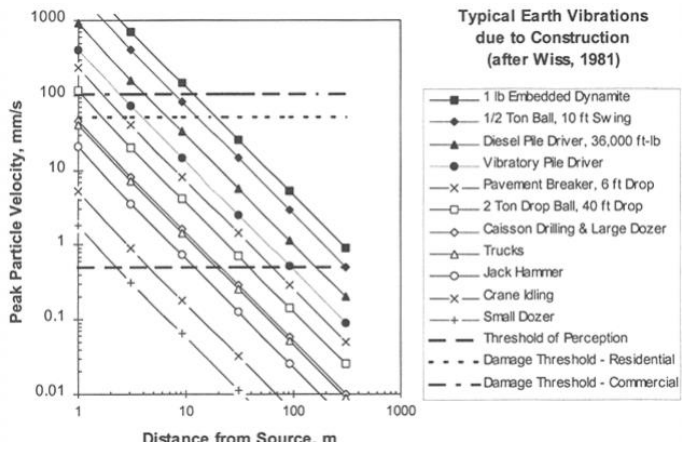

Fig. 4 Las vibraciones de la construcción en función de la distancia, por Hal Amick [9]

Por otro lado, el autor Abdul Karim, ver [10], habla acerca de la respuesta espectral relativa del suelo $\left(E_{\mathrm{f}}\right)$ y sobre la importancia que tiene la desviación $(\sigma)$ en este tema, para esto el usa dos ecuaciones como herramienta, para ver, medir y evaluar el comportamiento del suelo respecto a un estímulo, como puede ser el producido por una máquina para pilotaje, una vibro compactadora, tráfico de vehículos pesados o tráfico de trenes, las ecuaciones se pueden apreciar en la figura 5 .

$$
\sigma=\frac{\sum_{i=1}^{n}\left|\frac{E_{f i}(\text { source })-E_{f i}(\text { site })}{E_{f i}(\text { site })}\right|}{n} \quad E_{f}=\frac{\int_{f-1}^{f+1} R(f) d f}{\int_{f_{\min }}^{f_{\max }} R(f) d f}
$$

Fig. 5 Ecuaciones de desviación y de Respuesta espectral relativa del suelo, por Abdul Karim [10]. 
Como resultado de lo anteriormente establecido por el autor, se tiene que entre más se acerque a 0 el valor de desviación $(\sigma)$ mayor será la coincidencia entre el espectro del suelo y el producido por algún equipo de construcción, tal como se puede observar en la Figura 6, donde se evidencia que las curvas son similares y se cruzan en algunas frecuencias, el que ocurra esta coincidencia es un gran riesgo ya que puede generar un sin número de problemáticas ya sea directamente al suelo donde se está trabajando o al de zonas cercanas donde pueden haber estructuras y salir afectadas.

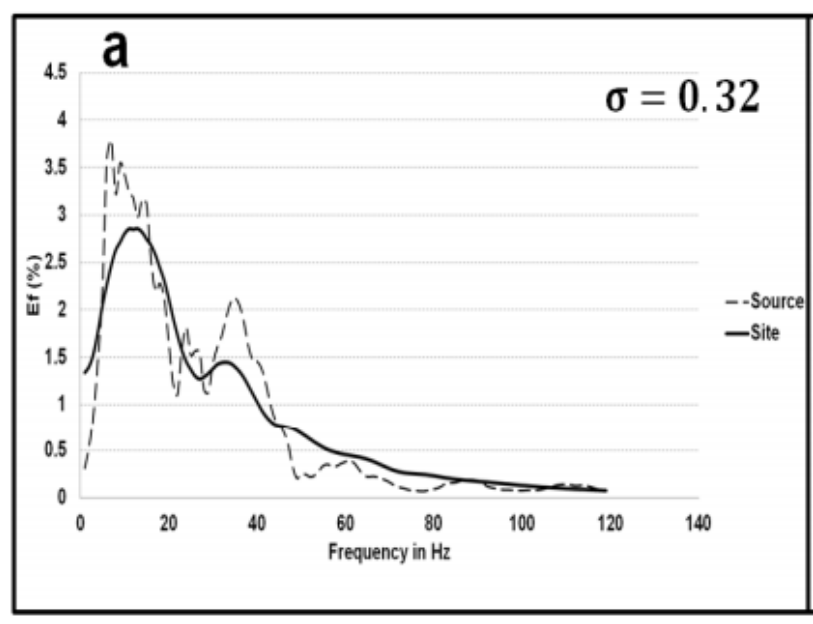

Fig. 6 Coincidencia espectral entre un registro de pilotajes y un suelo de $V S 3=100 \mathrm{~m} / \mathrm{s}$ y $B 10=0.2$, por Abdul Karim [10].

Cabe resaltar que el autor plantea que cuando se trata del espectro producido por máquinas para pilotaje, entre mayor sea la dureza del suelo, es decir una roca, mayor será el valor que se obtendrá de la desviación $(\sigma)$ por ende el riesgo será menor, mientras que con suelos más blandos como arcillas o arenas el valor de desviación tendera a ser cercano a 0 y por esto habrá un riesgo significativo. A continuación, en la figura 7 se puede observar algunos comportamientos espectrales registrados a maquinas piloteadoras [10].

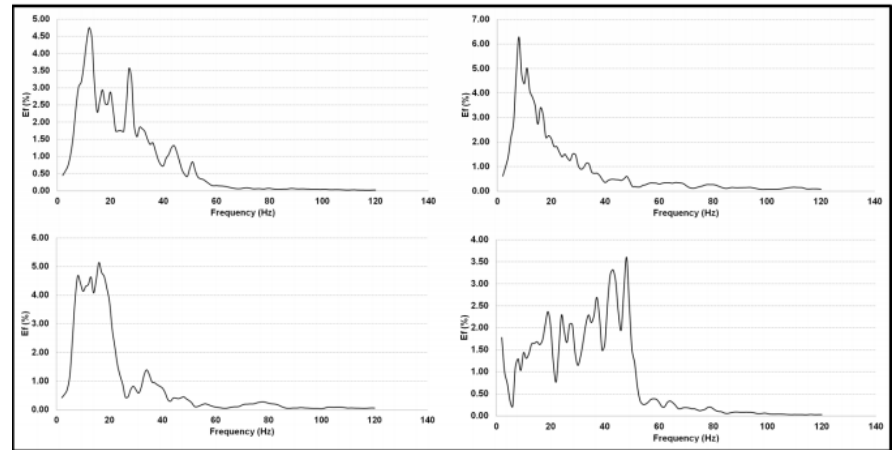

Fig. 7 Distribución relativa de la amplitud por frecuencia para varios registros de hinca de pilotes, por Abdul Karim [10].

En cuanto a los vibrocompactadores se refieren a equipos que generan varios ciclos de frecuencia que se encuentran entre 40 y $75 \mathrm{~Hz}$, estos afectan en especial a suelos que estén entre Vs=100 y $400 \mathrm{~m} / \mathrm{s}$, así mismo si la desviación es muy baja puede ocurrir grandes problemáticas, en la siguiente Figura se pueden ver algunas registros del espectro producido por este tipo de maquinaria [10].

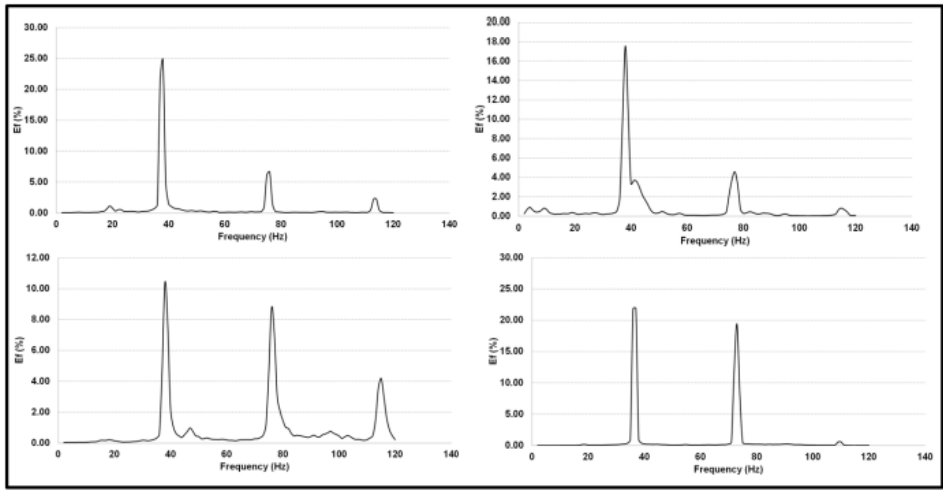

Fig. 8 Distribución relativa de la amplitud por frecuencia para diversas grabaciones de vibrocompactadores, por Abdul Karim [10].

En la investigación hecha por Hurtado, ver [11], la cual trata sobre un estudio comparativo de las metodologías para el análisis de cimentación de maquinarias. Este trabajo presenta un análisis comparativo de metodologías presentadas en la literatura de dinámica de suelos, empleadas en el estudio de cimentaciones sometidas a acciones vibratoria. Estas vibraciones fueron implementadas en un programa de cómputo, con el cual se realizaron diversos análisis para determinar las dimensiones apropiadas para la cimentación de los generadores de algunas centrales térmicas. Asimismo, en ese artículo se evaluó la variación de la respuesta del sistema vibratorio ante los cambios de magnitud del módulo de corte del suelo. La diferencia fundamental entre los métodos codificados radica en considerar las propiedades del sistema compuesto por la máquina, la cimentación y el suelo, como independientes o dependientes de la frecuencia de excitación, para fines de cálculo. Como producto de ese trabajo, se desarrolló una secuencia para el estudio de las cimentaciones superficiales ante acciones vibratorias, además el autor concluyo que las vibraciones admisibles usualmente son determinadas en función a la frecuencia de excitación.

Así mismo, en [12], se investigó la estimación del riesgo de daño debido a vibraciones inducidas por maquinaria en la ciudad de Cali, en dicho documento se evidencia distintas problemáticas en viviendas que se encuentran cercanas a una construcción, en el documento recalcan que la acción de las vibraciones generadas por la maquinaria de construcción sobre las edificaciones son una gran problemática, debido a que la maquinaria pesada genera vibraciones que pueden ser percibidas en edificaciones cercanas. En esa investigación se buscaba determinar la incidencia del equipo como un agente excitador de las viviendas cercanas a las obras civiles, para esto se tuvo en cuenta aspectos específicos como el tipo de máquina, el área de intervención y el tipo de construcción, además, los autores presentaron un análisis de la estimación del riesgo de daño debido a las vibraciones, donde se pudo analizar correctamente la incidencia de la distancia de la maquinaria a la vivienda, de esta forma se pudieron evaluar los posibles riesgos que a los que se verían sometidas las edificaciones cercanas.

En los últimos 5 años se han adelantado en la sabana de Bogotá distintas obras de construcción de acuerdo con el desarrollo territorial que se compone principalmente por la industria, el comercio, zonas residenciales, entre otros. De acuerdo con un estudio realizado por la Alcaldía de Bogotá, Figura 9, se tiene que el $12.1 \%$ de las obras realizadas hacen parte de los proyectos de hoteles, hospitales, universidades, colegios, iglesias, administración pública, parques y recreación; el $15.3 \%$ hace parte de las construcciones destinadas al comercio de la región tales como centros comerciales (Fontanar), centrales abastecimiento (PriceSmart), entre otros. Por otro lado, se tiene un $38.5 \%$ y un $32.8 \%$ en la construcción de industria y bodegas 
(Zona franca) respectivamente, esto debido a que estos son los dos ejes principales de la economía actual de la sabana de Bogotá, convirtiéndolo en una zona con un gran potencial económico en el país. Finalmente se tiene un $1.3 \%$ de oficinas, debido a que las oficinas son construidas mayoritariamente dentro de la ciudad de Bogotá [13].

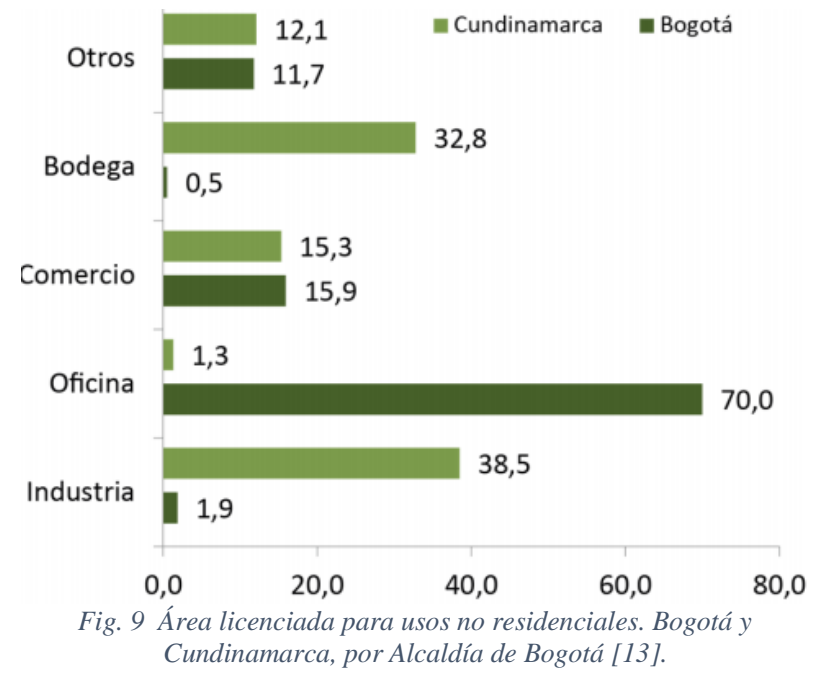

El suelo de la ciudad de Bogotá ha sido objeto de múltiples estudios que pretenden determinar su respuesta dinámica, algunos de los más relevantes han sido desarrollados por diferentes universidades bogotanas por medio de investigaciones sobre el comportamiento dinámico de los suelos presentes en la ciudad y su sabana, la Universidad Javeriana ha desarrollado estudios mediante ensayos Down Hole y triaxiales con suelos presentes en la zona 2 y 3 de la microzonificación sísmica de la ciudad, la Universidad Santo Tomás ha realizado ensayos de columna resonante para analizar propiedades dinámicas dentro del rango elástico de arcillas de origen lacustre presentes en la sabana occidente encontrando una relación entre los valores de confinamiento y módulo G, por su parte la Universidad Gran Colombia desarrolló un modelo constitutivo no lineal que define el comportamiento de arcillas blandas presentes en el campus de la Universidad Nacional de Colombia, otro estudio de la Universidad de los Andes al igual que la Universidad Javeriana realizo un aporte a la microzonificación sísmica de Bogotá por medio de la determinación del comportamiento de arcillas presentes en el suelo de la ciudad con el fin de observar los efectos de la velocidad de deformación en la degradación del módulo de rigidez y amortiguamiento, ofreciendo metodologías diferentes al modelo lineal equivalente utilizado en el programa Shake, estos estudios están soportados y se complementan con la teoría de diferentes autores mencionados en este artículo, y hacen parte de al menos 30 estudios de respuesta de dinámica local realizados en la ciudad de Bogotá.

\section{Caracterización de la zona de estudio}

\section{A. Localización}

La zona de estudio se encuentra ubicada en el municipio de Tenjo, Cundinamarca, Colombia cercana a la vía que de este municipio conduce a la ciudad de Bogotá D.C., accediendo a ella por la calle 80 o autopista Medellín. Específicamente la zona de estudio se localiza en la intersección vial de dos vías terciarias, las cuales comunican la zona rural del municipio de Tenjo con la vía principal Tenjo-Bogotá, aproximadamente sobre el $\mathrm{Km}$ 7. En la Figura 10 se presenta la localización del sitio.

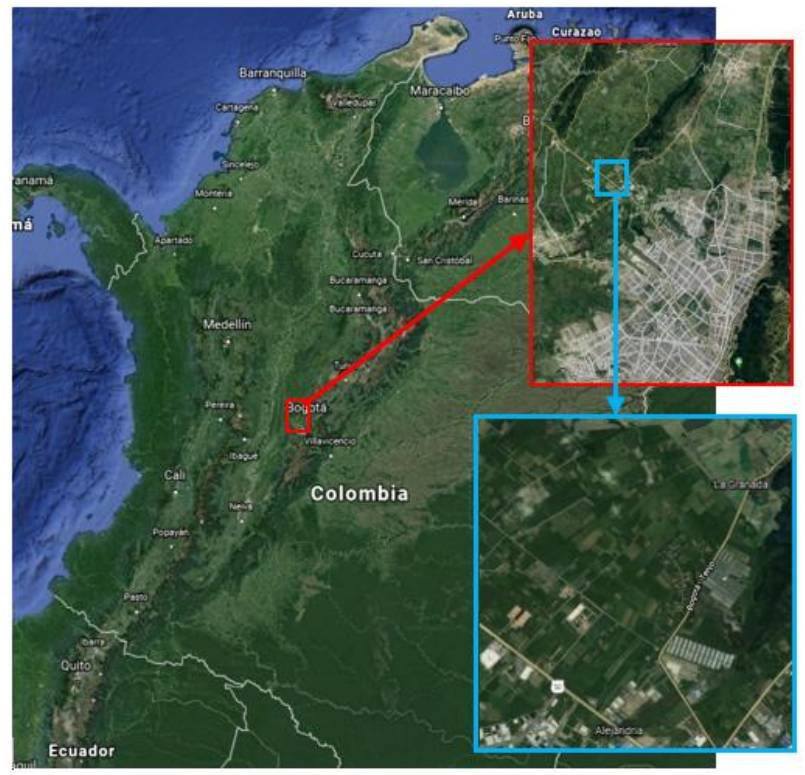

Fig. 10 Ubicación geográfica zona de estudio, Google Earth.

\section{B. Geología}

Según el Servicio Geológico Colombiano (SGC), en la zona del proyecto se presentan depósitos de Diatomeas conformados por arcillas, turbas y arcillas arenosas con niveles delgados de gravas. En la Figura 11 se presenta el entorno geológico de la zona de estudio donde es posible identificar un depósito "Q1-1" correspondiente al mencionado, rodeado por depósitos del tipo "k1kó-Stm" que contienen Shales, Calizas, Fosforitas y cuarzo arenitas y depósitos del tipo "Qca” denominados como Abanicos aluviales y depósitos coluviales.

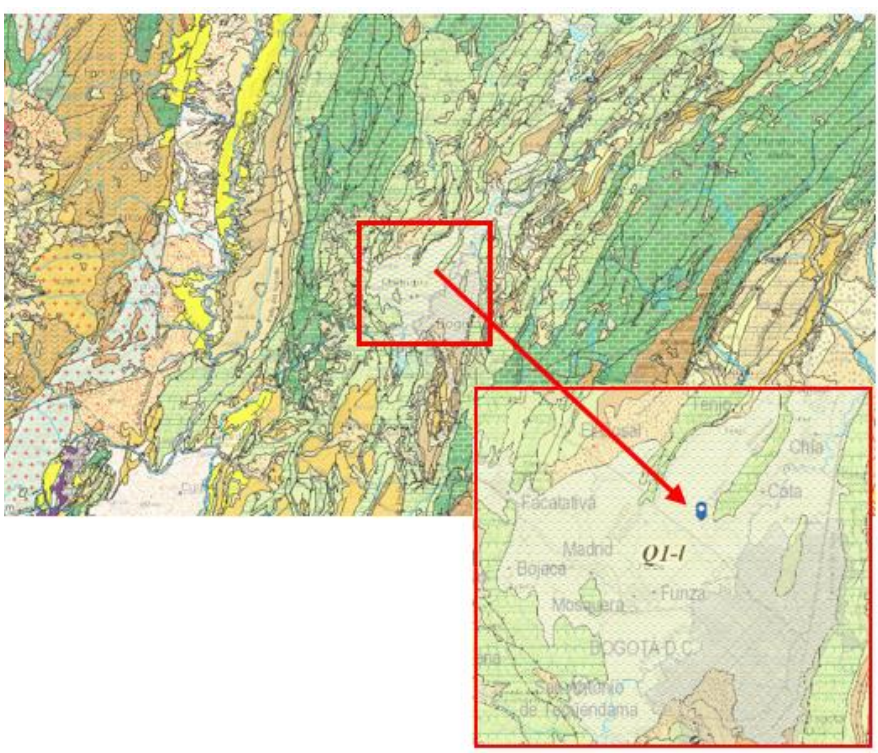

Fig. 11 Entorno geológico de la zona de estudio. Por Gómez [14].

\section{Geotecnia}

Con respecto a la clasificación geotécnica de los depósitos, se consultó el decreto de microzonificación sísmica de Bogotá, del cual se extraen las figuras $12,13,14,15$ y 16 . 


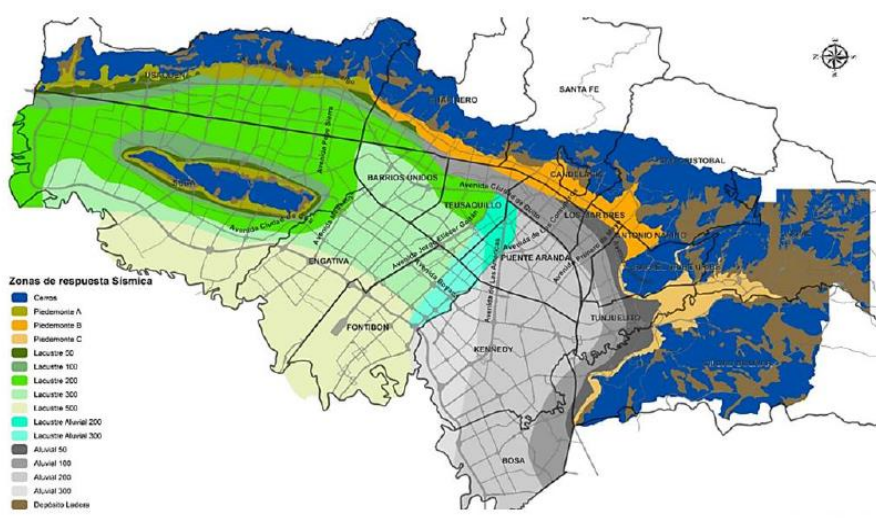

Fig. 12 Zonificación de la respuesta sísmica de los suelos de Bogotá D.C., por IDIGER [15].
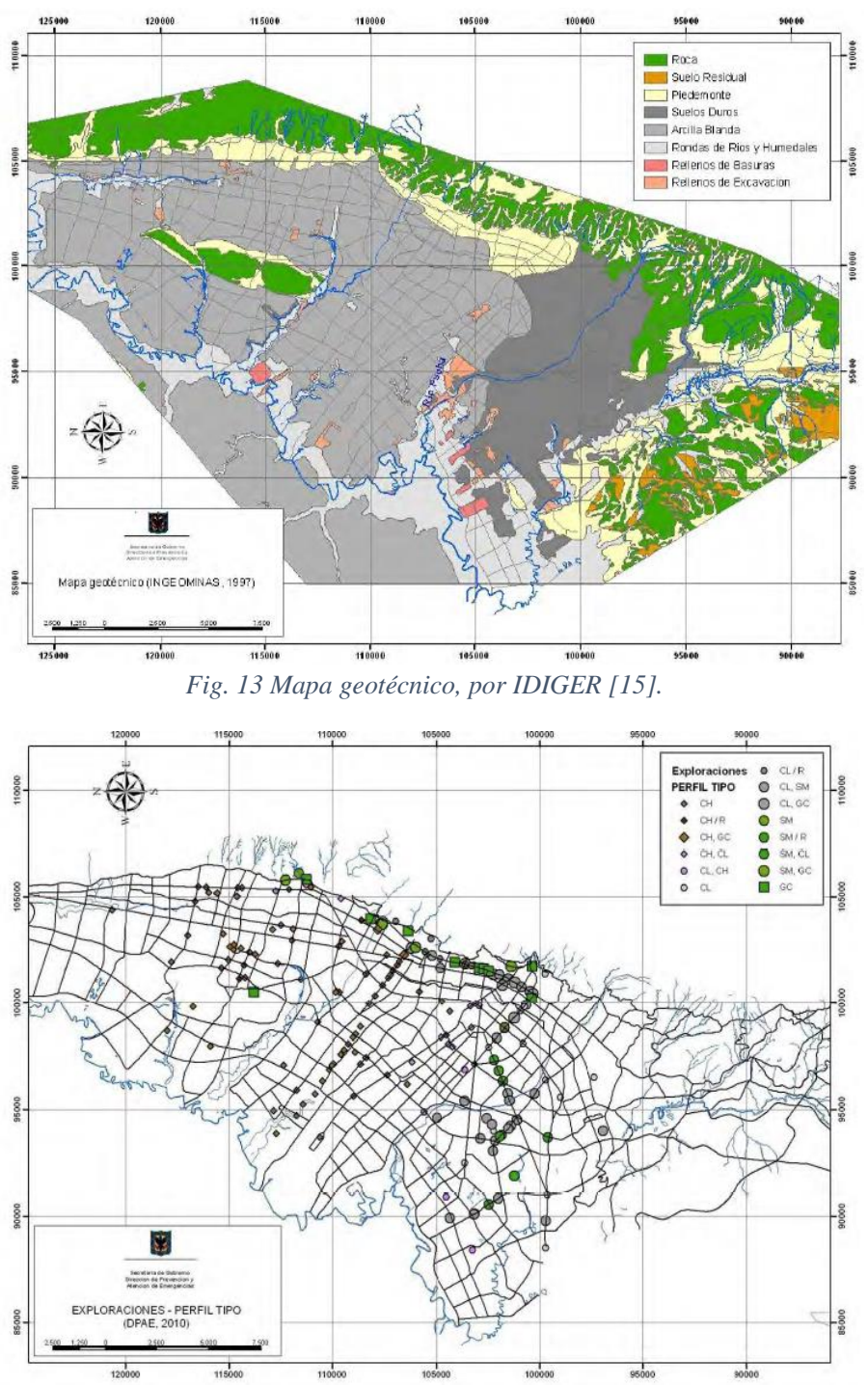

Fig. 14 Mapa de perfiles típicos superficiales, por IDIGER [15].

Teniendo en cuenta la ubicación de la zona de estudio respecto a su cercanía con el límite occidental de la ciudad de Bogotá D.C. y con base en que esta también se encuentra clasificada como tipo de suelo "Q1-1" es posible asumir para la zona de estudio los parámetros de un suelo lacustre 500, el cual según el decreto 523 de 2010 corresponde a un suelo blando de baja a media capacidad portante y muy compresible conformado según los mapas de "Respuesta sísmica", "Geotecnia” y “
"Perfiles típicos superficiales" [15], principalmente por arcillas blandas de alta plasticidad $(\boldsymbol{C H})$ que pueden ser arcillas limosas y/o arcillas arenosas con sectores de lentes de turba.

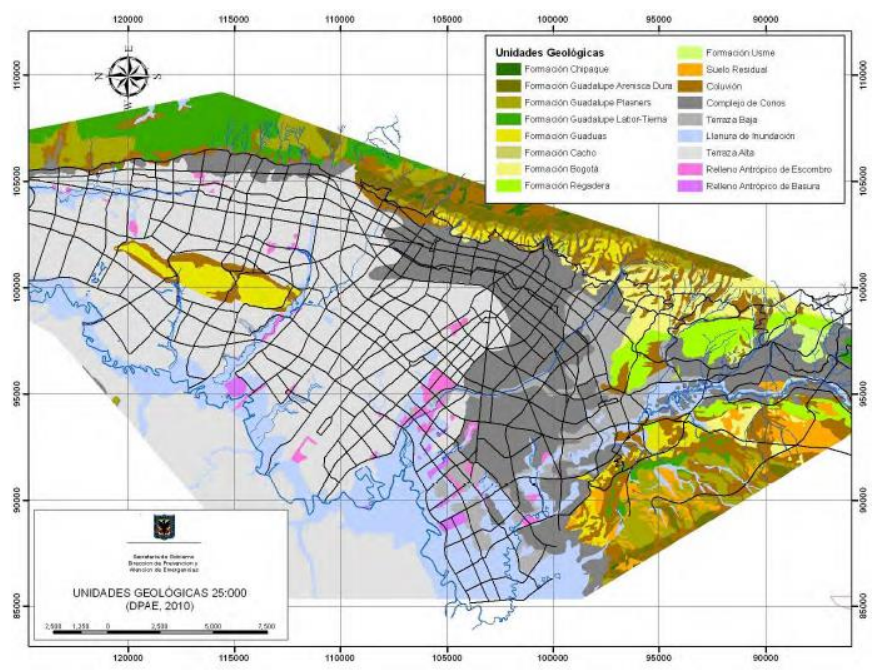

Fig. 15 Mapa geológico INGEOMINAS 1995, por IDIGER [15].

Según el mapa geológico de INEGOMINAS de la ciudad de Bogotá, se puede observar que hacia la sabana occidente de la ciudad se encuentran terrazas altas y llanuras de inundación en la ronda del rio Bogotá, por lo cual teniendo en cuenta la ubicación de las perforaciones podría asumirse la misma como una terraza alta producto de un depósito aluvial.

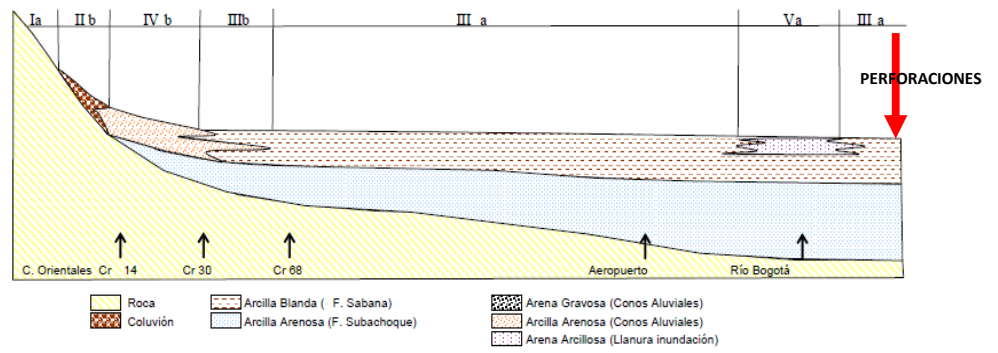

Fig. 16 Esquema geotécnico típico de una sección este-oeste entre cl 13 a cl 127 aprox, por IDIGER [15].

Tomando como referencia la calle 80 por ser el punto más cercano de la ciudad de Bogotá a la zona de estudio se puede apreciar que a medida que se avanza hacia la sabana occidente se profundiza mucho más el estrato rocoso tomando mayor predominancia en el subsuelo la "Formación Sabana" compuesta por arcilla blanda que se encuentra a nivel superficial y la "Formación Subachoque" compuesta por arcilla arenosa que se encuentra por debajo de los estratos de la "Formación Sabana".

\section{Respuesta sísmica de los depósitos}

Teniendo en cuenta el enfoque principal del presente artículo el cual es analizar y estudiar la respuesta del suelo ante fuerzas dinámicas se hace necesaria la caracterización sísmica de la zona de estudio. Se consultó la norma sismo resistente colombiana Nsr-10, de la cual se extraen, la figura 17 y las tablas 1 y 2 . 


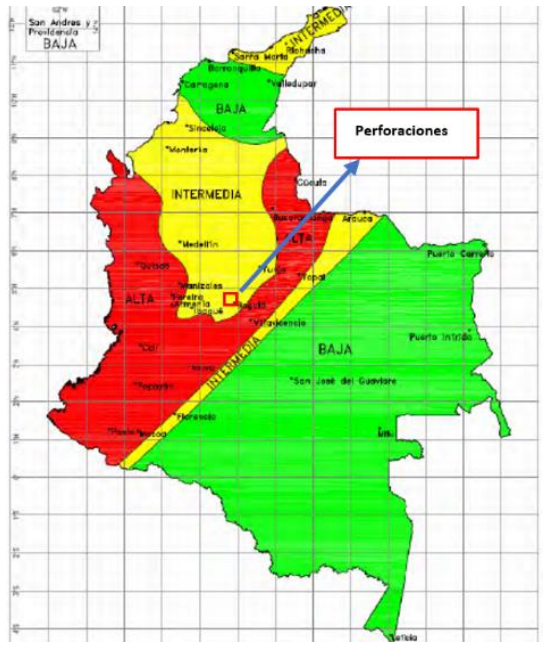

Fig. 17 Mapa de zonificación sísmica de Colombia, por IDIGER [15].

Tabla 1. Valor de Aa y de Av para las ciudades capitales de departamento; Tabla A.2.3-2, NSR-10 [16]

\begin{tabular}{|c|c|c|c|}
\hline Ciudad & $\mathbf{A}_{\mathbf{a}}$ & $\mathbf{A}_{\mathbf{v}}$ & $\begin{array}{c}\text { Zona de } \\
\text { Amenaza } \\
\text { Sísmica }\end{array}$ \\
\hline Arauca & 0.15 & 0.15 & Intermedia \\
\hline Armenia & 0.25 & 0.25 & Alta \\
\hline Barranquilla & 0.10 & 0.10 & Baja \\
\hline Bogotá D. C. & 0.15 & 0.20 & Intermedia \\
\hline
\end{tabular}

Tabla 2. Valores de Fa, Fv, Aa y Av para el tipo de suelo en estudio, por Alcaldía Mayor de Bogotá [17].

\begin{tabular}{|c|c|c|c|}
\hline FA & FV & Aa & Av \\
\hline 2,1 & 3,2 & 0,15 & 0,20 \\
\hline
\end{tabular}

Con base en la gráfica zonal de amenaza sísmica presente en el titulo A de la NSR-10 se puede evidenciar que la zona de estudio se encuentra en una zona de amenaza sísmica intermedia, lo cual indica la posibilidad de presencia de sismos de magnitudes medias y repetitivas, el valor de coeficiente de aceleración horizontal de pico efectiva ( $\mathrm{Aa}$ ) tiene un valor de 0.15 y el coeficiente que representa la velocidad horizontal de pico efectiva (Av) tiene un valor de 0.20 [16].

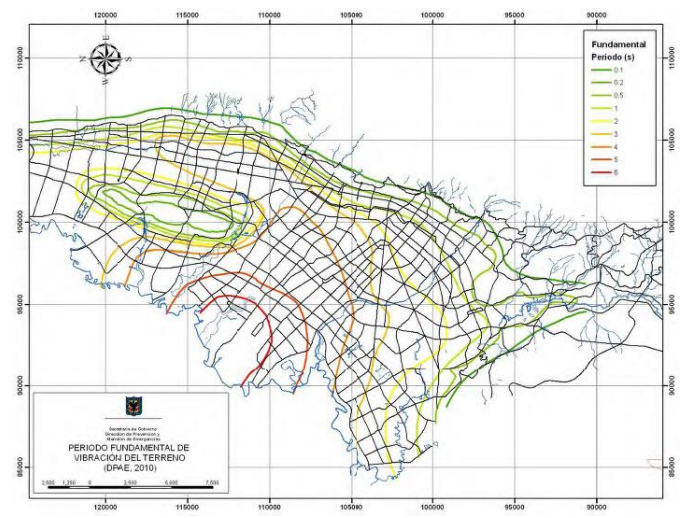

Fig. 18 Gráfica periodo fundamental de vibración del terreno, por IDIGER [15].

A través de la gráfica de periodo fundamental de vibración del terreno se observa que la zona limítrofe occidental de la ciudad de Bogotá sobre la calle 80, tomada como punto de referencia de la zona de estudio presenta un periodo que oscila entre 4.5 y $6.5 \mathrm{~s}$.
Así mismo el decreto 523 de 2010 indica que este tipo de suelo presenta velocidades de onda promedio $50 \mathrm{~m}$ Vs $<175 \mathrm{~m} / \mathrm{s}$ y humedad superior al $80 \%$.

Tabla 3 Descripción de las zonas geotécnicas para respuesta sísmica, por IDIGER [15].

\begin{tabular}{|c|c|c|c|c|c|}
\hline Nombre & Geotecnla & 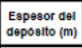 & \begin{tabular}{|l|} 
Perlodo \\
fundamantal \\
del avelo (o)
\end{tabular} & Goologis & Geomortologta \\
\hline \multirow{7}{*}{ Lacustre A } & \multirow{7}{*}{ Sueto lacustre muy olando } & $<50$ & $1.0-1.5$ & & \multirow{16}{*}{$\begin{array}{l}\text { Planicle } \\
\text { plats }\end{array}$} \\
\hline & & 50-100 & $1.5-2.5$ & & \\
\hline & & $100-150$ & $2.5-3.0$ & & \\
\hline & & $150-200$ & $3.0-3.5$ & & \\
\hline & & $200-250$ & $3.5-4.0$ & & \\
\hline & & $250-300$ & $4.0-4.5$ & & \\
\hline & & $300-400$ & $4.5-5.5$ & & \\
\hline \multirow{4}{*}{ Lacustre B } & \multirow{4}{*}{ Sueto lacustre blando } & $100-150$ & 23.2 .85 & Temraza Ata - Lacustre & \\
\hline & & $150-200$ & $2.8 \cdot 3.3$ & & \\
\hline & & $200-250$ & $3.3-3.8$ & & \\
\hline & & $250-300$ & 3.8 .4 .3 & & \\
\hline \multirow{5}{*}{ Lacustre C } & \multirow{5}{*}{ Suelo lacustre - auvial } & $100-150$ & $2,3-2.8$ & & \\
\hline & & $150-200$ & $2.8-3.3$ & & \\
\hline & & $200-250$ & $3.3-3.8$ & & \\
\hline & & $250-300$ & $3.8-4.3$ & & \\
\hline & & $300-400$ & $4.3-5.3$ & & \\
\hline
\end{tabular}

Como se puede observar en la tabla 3 , asumiendo la clasificación de la zona como "Lacustre A 500", se tiene un periodo fundamental del suelo que está entre 5,5 y 6,5s. Concordando con la gráfica de periodo fundamental y permitiendo para esta investigación asumir un valor de periodo fundamental del suelo entre 5,5 y $6,5 \mathrm{~s}$.

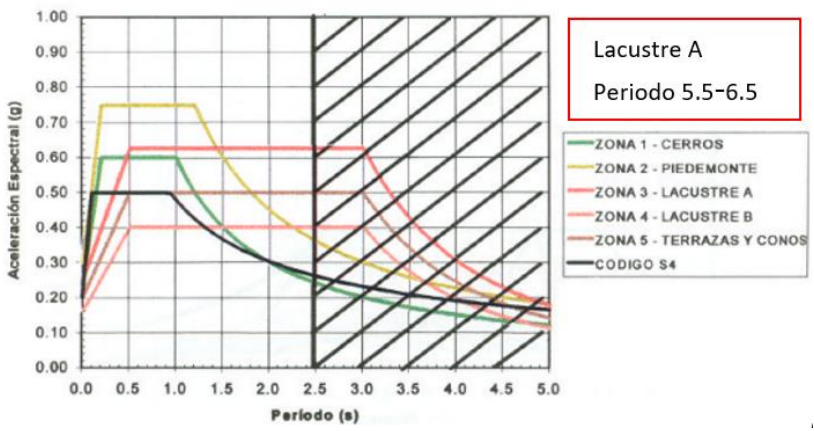

Fig. 19 Gráfica de espectro, por INGEOMINAS [18]

Con base en la gráfica de espectro se determina que el suelo se manifiesta por medio de una respuesta intermedia, con grandes oscilaciones y un amplio periodo lo que se traduce en una reacción menos violenta y una disipación de energía más lenta. Sin embargo, debido a las grandes oscilaciones la percepción en el movimiento puede llegar a ser muy fuerte, ocasionando daños moderados a las estructuras con un PGA\% entre 18-34, según el mapa de amenaza sísmica del Servicio Geológico Colombiano. Como se muestra en la Figura 19.

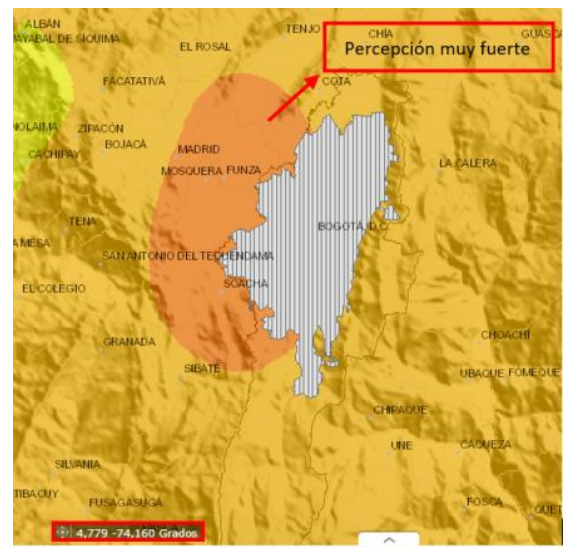

Fig. 20 Mapa de amenaza sísmica, Servicio Geológico Colombiano. 
ESPECTRO DE DISEÑO NSR - 10

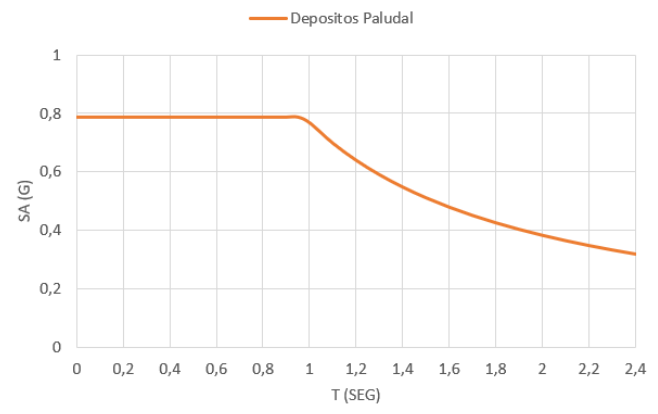

Fig. 21 Grafica de espectro de diseño para Deposito Paludal

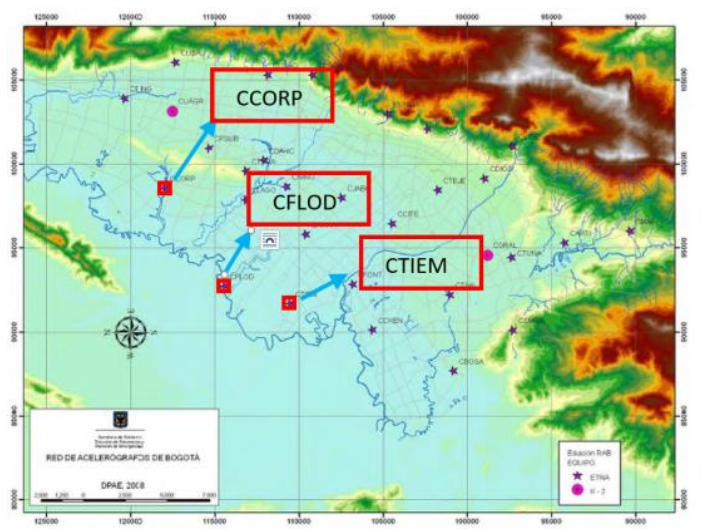

Fig. 22 Localización de estaciones de la RAB, por IDIGER [15].

La RAB (Red de Acelerógrafos de Bogotá) cuenta con 30 estaciones distribuidas en el territorio distrital. Sobre la zona limítrofe occidental se encuentran las estaciones Universidad Corpas (CCORP), Parque la florida (CFLOD), y Club El Tiempo (CTIEM), las cuales se encuentran instaladas sobre zonas geológicas con características similares a la zona estudiada en el presente artículo.

A continuación, se presentan en la tabla 4 los registros medidos para diferentes parámetros sísmicos importantes en el análisis dinámico de los suelos a partir del sismo Quetame, ocurrido el 24 de mayo del 2008 con una magnitud de 5.7 en la escala Richter y epicentro en Quetame, Cundinamarca, El cuál para esa fecha había presentado las mayores aceleraciones desde la implementación de la RAB.

Tabla 4 Parámetros sísmicos de los registros de sismo Quetame en Bogotá, por IDIGER [15].

\begin{tabular}{|c|c|c|c|c|c|c|c|c|}
\hline Código & Nombre & $\begin{array}{c}\text { Epicentro } \\
(\mathrm{Km})\end{array}$ & $\begin{array}{c}\text { Hipocentro } \\
(\mathrm{Km})\end{array}$ & $\begin{array}{l}\text { Intensidad } \\
\text { Arias }(\mathrm{cm} / \mathrm{s})\end{array}$ & $\begin{array}{c}\text { Fase } \\
\text { intensa }(\mathrm{s})\end{array}$ & $\begin{array}{c}\text { Amax } \\
\left(\mathrm{cm} / \mathrm{s}^{2}\right)\end{array}$ & $\begin{array}{c}\text { Periodo } \\
\text { Natural }(\mathrm{s})\end{array}$ & $\begin{array}{l}\text { Profundidad } \\
\text { Deposito }(\mathrm{m})\end{array}$ \\
\hline
\end{tabular}

\begin{tabular}{|c|c|c|c|c|c|c|c|}
\hline \begin{tabular}{|l|l|} 
CTIEM & CLUBEL TIEMPO \\
\end{tabular} & 49.7 & 49.9 & 28 & $32.6^{*}$ & 26.8 & 3.25 & 375 \\
\hline \begin{tabular}{l|l|l|l|l|} 
ORP & UNIVESIDAD CORPAS \\
\end{tabular} & 49.9 & 50.1 & 22 & 69.8 & 16.0 & 2.7 & 220 \\
\hline PARQUELAFLORIDA & 51.8 & 52 & 2.7 & $52.9^{*}$ & 18.5 & 4.25 & 500 \\
\hline
\end{tabular}

\section{CARACTERISTICAS DEL PERFIL ESTRATIGRAFICO}

\section{E. Exploración del subsuelo}

Para conocer el tipo de suelo presente en la zona de estudio se realizaron dos sondeos en la zona de estudio con una profundidad máxima de 5,80m y 6,00m mediante el uso de SPT y tubería de pared delgada "Shelby", de las cuales fueron recuperadas 4 y 5 muestras respectivamente a diferentes profundidades como se relaciona en las ilustraciones 4 y 5 , posteriormente fueron enviadas al laboratorio para realizar la identificación del tipo de suelo objeto de estudio y sus características tanto físicas como mecánicas para su correspondiente análisis de caracterización y clasificación, con el fin de obtener la información pertinente que permita la realización de un perfil estratigráfico de la zona estudiada. En la siguiente Figura se presenta la localización de las perforaciones.

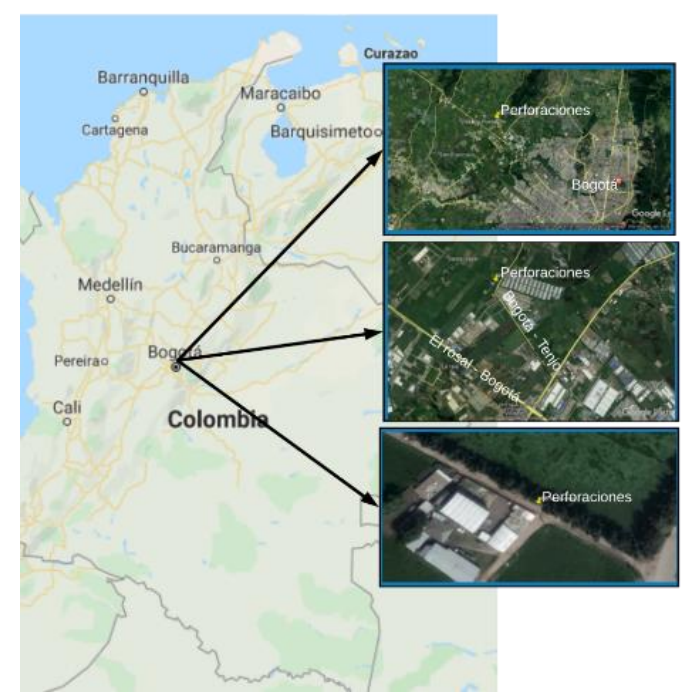

Fig. 23 Localización de las perforaciones, Google Earth.

El punto de referencia para la extracción de muestras obedece a las coordenadas de latitud 4.777719 y longitud -74.161323 .

\section{F. Ensayos de laboratorio}

Sobre las muestras de suelo extraídas se llevó a cabo un programa de exploración del subsuelo con el fin de realizar una caracterización física y mecánica del suelo trabajado, el cual incluyó ensayos de Peso unitario, gravedad específica, hidrometría, límites, compresión inconfinada y consolidación, cuyos resultados se presentan en tabla 5.

Tabla 5 Resultados de ensayos de caracterización

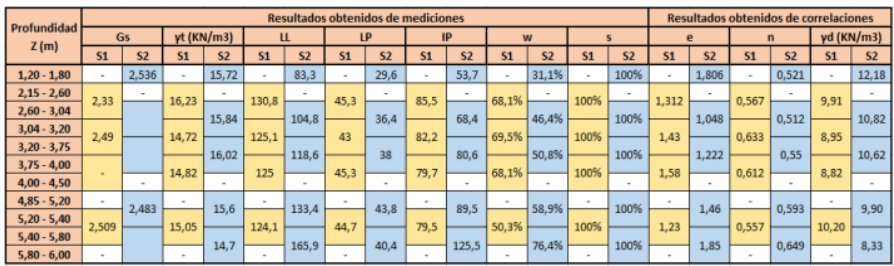

\section{G. Perfil estratigráfico promedio}

Como resultado del análisis de los datos obtenidos a partir de ensayos de laboratorio de gravedad específica (Gs), peso unitario (ysat) y límites de Atterberg, se establece una caracterización promedio preliminar del tipo de suelo trabajado, la cual basada en la literatura de bardet corresponde a una fat clay soft, $(\mathrm{CH})$ con bajos contenidos de limos y arena fina, donde se evidencia presencia de minerales como illita, caolinita y montmorillonita además de feldespatos potásicos, serpentinas y halloysita

A continuación, se presenta la tabla 6 la cual contiene los resultados de laboratorio unificados para un perfil estratigráfico promedio. 


\begin{tabular}{|c|c|c|c|c|c|c|c|c|c|c|c|}
\hline \multirow{2}{*}{$\begin{array}{c}\text { Profundidad } \\
\mathrm{Z}(\mathrm{m})\end{array}$} & & \multicolumn{10}{|c|}{ Propiedades del perfil estratigráfico } \\
\hline & & Gs & pt & LL & LP & IP & w & $\mathrm{s}$ & $\mathbf{e}$ & $\mathrm{n}$ & pd \\
\hline $1,20-1,80$ & 1,50 & 2,536 & 5,72 & & & 3,7 & & 1,00 & 06 & 0,521 & 12,18 \\
\hline & & 2 & 1 & & & & & & & נו & \\
\hline 2, & 2,82 & 2,330 & 16,04 & 117,8 & & 7,0 & 73 & 00 & 80 & 540 & 10,37 \\
\hline 3, & 12 & 2,490 & 15,28 & 115 & 39 & 5,3 & 30 & 00 & 239 & 0,573 & 9,89 \\
\hline & 3 & 2,490 & 15,37 & & & & & & 26 & 92 & 79 \\
\hline & & & 15,42 & & & & & & & 0,581 & 72 \\
\hline & & & 70 & & & & & & & 0,612 & \\
\hline & & 48 & 15,60 & 133,4 & 138 & 895 & 39 & 0 & 50 & 0,593 & 0 \\
\hline 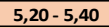 & 5, & 2,496 & 15,33 & 128,8 & 44, & 84 & 0,546 & 1,00 & 345 & 0,575 & 10,05 \\
\hline & 5 , & 2,509 & 14,88 & 145,0 & 42 & 5 & 0, & 1,00 & 40 & 0,603 & 9,27 \\
\hline $5,80-6,00$ & 5,90 & & 14,70 & 165,9 & 40,4 & 125,5 & 0,764 & 1,00 & 1,850 & 0,649 & 8,33 \\
\hline
\end{tabular}

En la figura 24 se observa el comportamiento del suelo y la variación de sus propiedades en función de la profundidad.
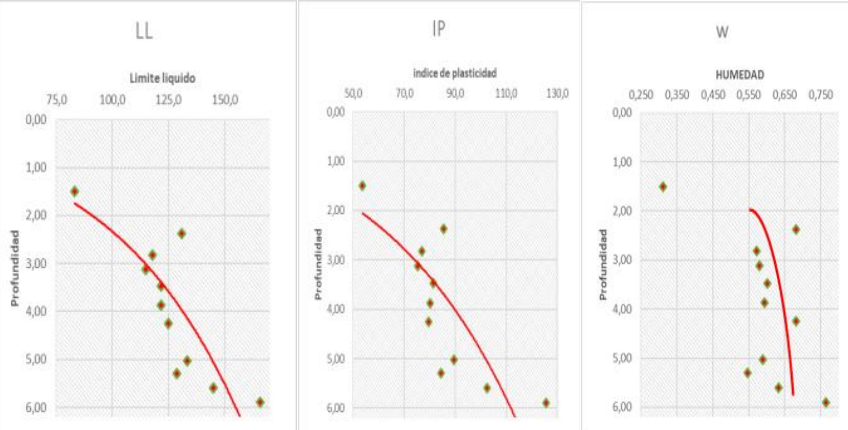

Fig. 24 Variación del contenido de agua del suelo con la profundidad.

Tal y como se contempla en la figura 24 , hay una tendencia al aumento del contenido de agua natural, el límite líquido y el índice de plasticidad con la profundidad, lo cual es un indicativo del aumento de la compresibilidad y la disminución de la consistencia, típico del perfil de suelos lacustres de la sabana de Bogotá. Se observan dos estratos, el primero alcanza una profundidad de hasta $2.2 \mathrm{~m}$, y registra una humedad baja. Subyaciendo, se encuentra un estrato notablemente más plástico, con una tendencia la variación que se presume constante a hasta la profundidad investigada.
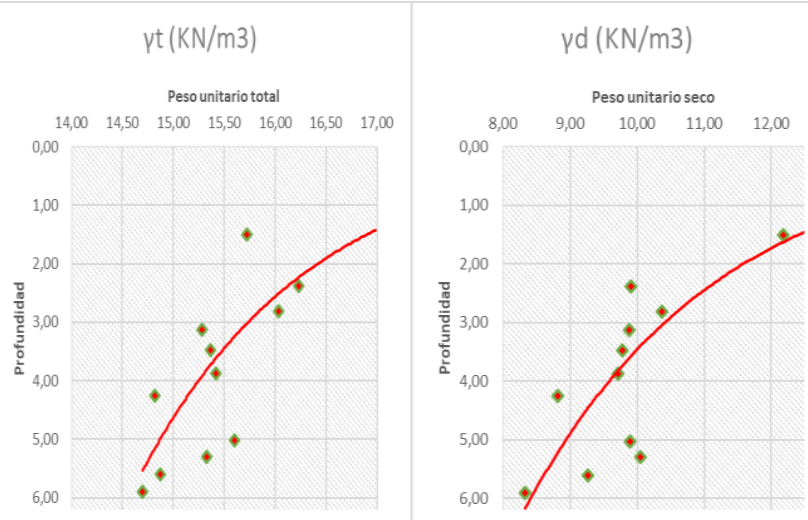

Fig. 25 Variación del peso específico total y seco en función de la profundidad.

En la figura 25, se puede observar que, conforme aumenta el valor de la profundidad se presenta una disminucion tanto en el peso especifico total como en el seco, lo que refleja una perdida de densidad del material y asi mismo, se evidencia que a mayor profundidad es posible encontrar que el material de suelo este mas suelto, lo cual contrasta con el aumento del contenido de agua en el suelo ya que a mayor profundidad el agua va a llenar mayor cantidad de vacios y al disiparse la presion de poros el peso seco tendera a ser menor.

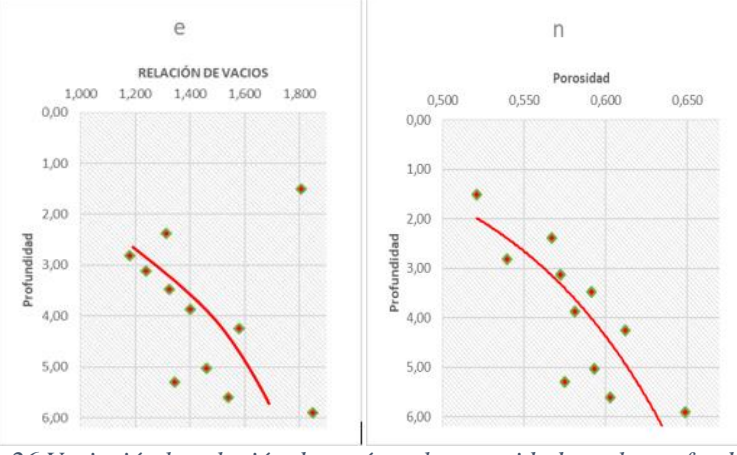

Fig. 26 Variación la relación de vacíos y la porosidad con la profundidad.

En la figura 26 se observa un aumento en la relación de los vacíos y la porosidad del material con la profundidad, lo cual es consecuente con lo obtenido para el análisis del contenido de agua y típico de los materiales en la zona estudiada, se observa nuevamente un cambio evidente para una profundidad de $2.50 \mathrm{~m}$, identificando entonces un cambio en las características estratigráficas del perfil. Las relaciones de vacíos obtenidas varían entre 1.20 y 1.80 lo cual es consecuente con lo obtenido por, ver [19], donde manejan un rango de 0.55 a 2.1 para una profundidad de 6 metros y es un indicativo de la compresibilidad de los materiales.

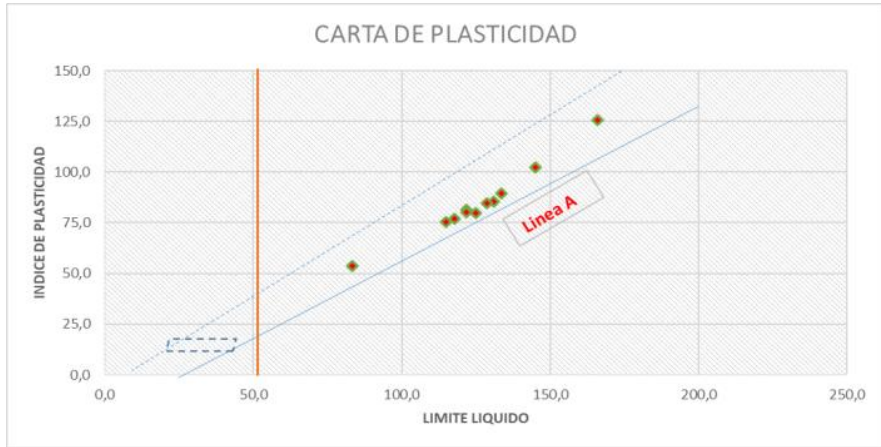

Fig. 27 Carta de plasticidad.

Como parte de la clasificación del suelo es importante realizar un análisis de consolidación y resistencia del material estudiado con el propósito llegar a la determinación del comportamiento mecánico del suelo bajo el efecto de cargas estáticas impuestas, es por esta razón que se toma para estos ensayos una muestra de cada sondeo para efectos de análisis de consolidación y una muestra del sondeo 1 junto a dos muestras del sondeo 2 para el ensayo de compresión inconfinada relacionadas en la tabla 7.

Tabla 7 Resultados de ensayos de resistencia perfil estratigráfico promedio.

\begin{tabular}{|c|c|c|c|c|c|c|c|c|c|c|c|}
\hline \multirow{3}{*}{$\begin{array}{c}\text { Profundidad } \\
\mathrm{Z}(\mathrm{m})\end{array}$} & \multirow{3}{*}{$z$} & \multicolumn{10}{|c|}{ Propiedades de resistencia del perfil estratigráfico } \\
\hline & & \multicolumn{6}{|c|}{ Consolidación } & \multicolumn{4}{|c|}{ Compresión inconfinada } \\
\hline & & Cc & Cs & $\mathrm{cu} \mathrm{m} 2 / \mathrm{s}$ & op Kpa & \begin{tabular}{|l|l}
$\sigma o \mathrm{Kpa}$ \\
\end{tabular} & Estado & qu Kpa & Сu Kpa & $\Phi$ & Consist \\
\hline $1,20-1,80$ & 1,50 & 0,198 & 0,053 & 0,0016 & 290,0 & 3,24 & SC & 542,30 & 271,640 & & Dura \\
\hline $2,15-2,60$ & 2,38 & 0,379 & 0,024 & 0,0035 & 280,0 & 5,54 & SC & & & & \\
\hline $2,60-3,04$ & 2,82 & 0,379 & 0,024 & 0,0035 & 280,0 & 5,54 & SC & & & & \\
\hline $3,04-3,20$ & 3,12 & & & & & & & & & & \\
\hline $3,20-3,75$ & 3,48 & & & & & & & & & & \\
\hline $3,75-4,00$ & 3,88 & & & & & & & 119,64 & 59,82 & $45^{\circ}$ & Firme \\
\hline $4,00-4,50$ & 4,25 & & & & & & & 119,64 & 59,82 & $45^{\circ}$ & Firme \\
\hline $4,85-5,20$ & 5,03 & & & & & & & 216,73 & 107,870 & $48^{\circ}$ & Muy firme \\
\hline $5,20-5,40$ & 5,30 & & & & & & & 190,74 & 95,13 & $55^{\circ}$ & Firme \\
\hline $5,40-5,80$ & 5,60 & & & & & & & 164,75 & 82,380 & $55^{\circ}$ & Firme \\
\hline $5,80-6,00$ & \begin{tabular}{|l|}
5,90 \\
\end{tabular} & & & & & & & & & & \\
\hline
\end{tabular}

Como resultado del ensayo de consolidación se puede apreciar un material $\mathrm{CH}$ sobre consolidado, el cual presenta un coeficiente de consolidación de $0.035 \mathrm{~m}^{2} / \mathrm{s}$ y un esfuerzo de pre consolidación que varía entre 280 y $290 \mathrm{kPa}$, por otro lado, el ensayo de compresión inconfinada arroja unos resultados, en los cuales se observa que la 
resistencia máxima promedio a la falla del suelo es de $162 \mathrm{kPa}$ y que la resistencia admisible promedio es de $81 \mathrm{kPa}$, además se puede conocer que la consistencia del suelo es firme en su mayoría y muy firme entre la profundidad de $4.85 \mathrm{~m}$ y $5.20 \mathrm{~m}$.

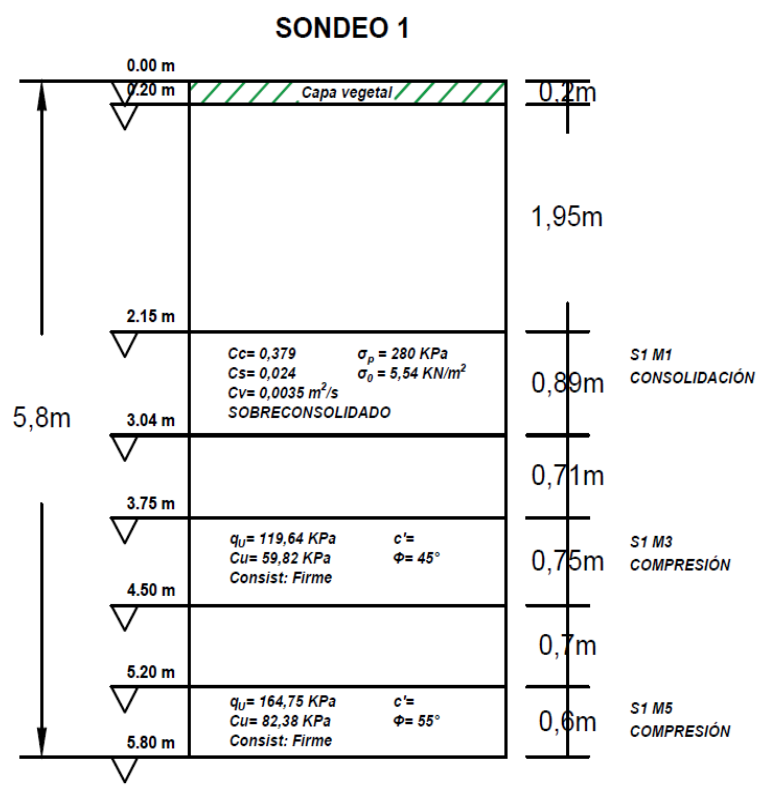

Fig. 28 Resultados de muestras sondeo 1 sometido a ensayos de consolidación y compresión inconfinada

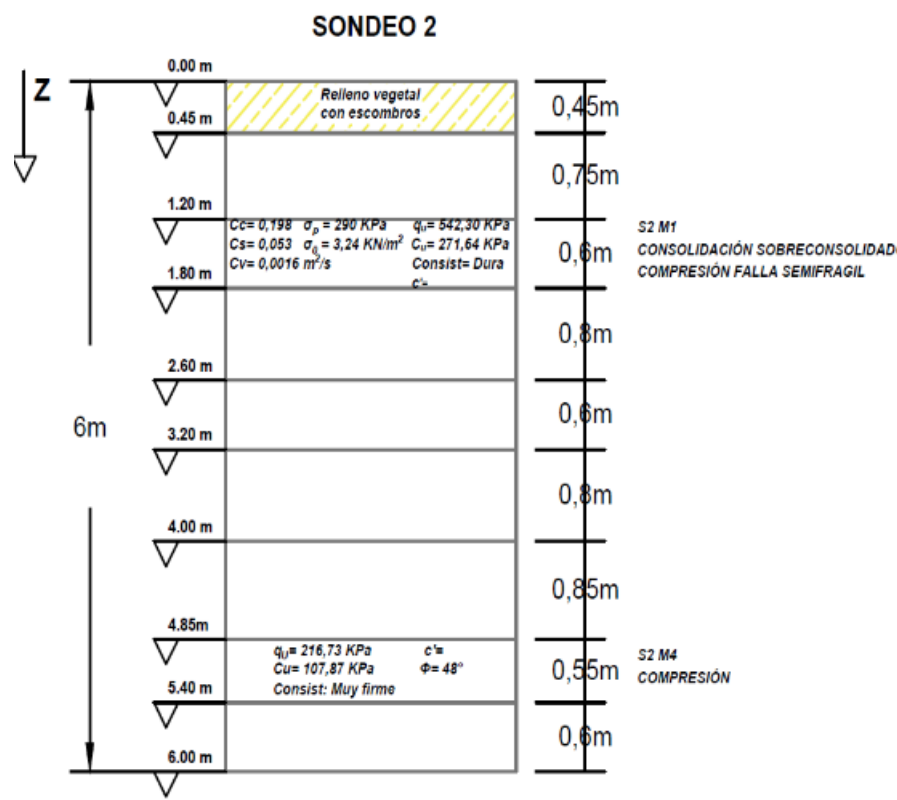

Fig. 29 Resultados de muestras sondeo 2 sometido a ensayos de consolidación y compresión inconfinada.

Como resultado del estudio de caracterización y clasificación del suelo se define como material analizado una Arcilla blanda húmeda de alta plasticidad (CH) (Fat clay soft) dado que su índice de plasticidad se encuentra en un rango ente 53,7 - 125,5 el cuál varía en función de la profundidad de la muestra, a pesar de ser una arcilla blanda presenta una consistencia dura a firme durante el ensayo de compresión inconfinada, arrojando valores de resistencia antes de la falla entre 120 y $540 \mathrm{Kpa}$, presentando tipo de falla frágil a cortante.

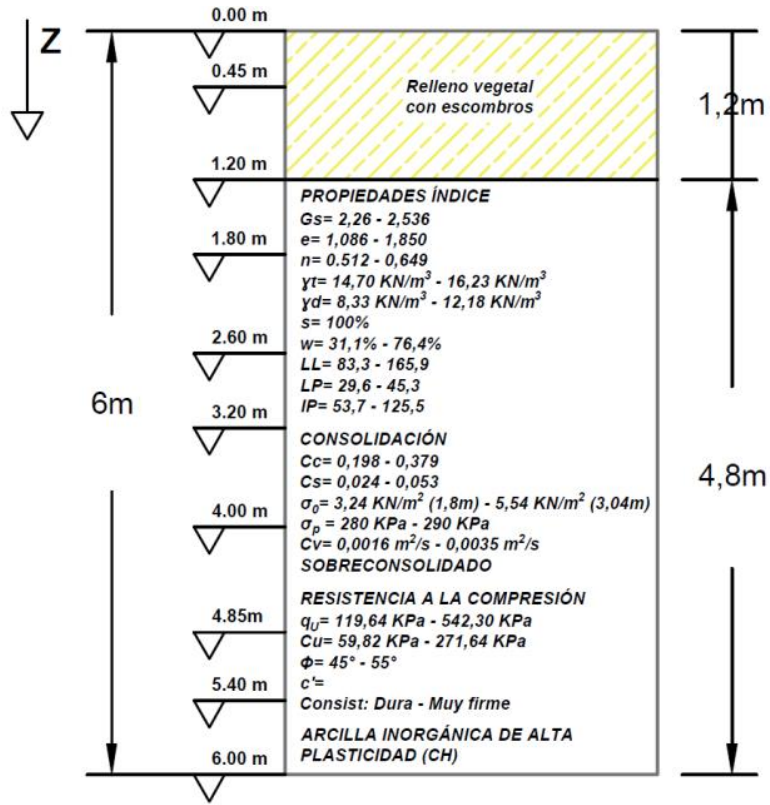

Fig. 30 Perfil estratigráfico promedio de la zona de estudio.

Mediante el perfil estratigráfico promedio establecido para la zona de estudio se definen dos estratos, el primero de ellos con profundidad de $1,20 \mathrm{~m}$ corresponde a material limo-arcilloso con contenido de materia vegetal y orgánica, por lo cual se decide descartarlo como objeto de estudio y se centra la investigación en el segundo estrato, definido como Arcilla blanda de alta plasticidad (CH) de 4,80m de espesor el cual se encuentra ubicado entre los $1,20 \mathrm{~m}$ y $\operatorname{los} 6,00 \mathrm{~m}$ de profundidad, dado que el porcentaje de saturación para todas las muestras analizadas es de $100 \%$ se asume un nivel freático por encima de $1,20 \mathrm{~m}$ posiblemente cercano a la superficie teniendo en cuenta una capa vegetal de $0,20 \mathrm{~m}$ de profundidad y presencia de materia orgánica y vegetal aproximadamente hasta $0,45 \mathrm{~m}$ de profundidad.

\section{ENSAYOS DE COLUMNA RESONANTE}

En un estudio realizado en la misma zona, ver [20] se encontró un suelo compuesto por arcillas blandas de alta plasticidad $(\mathrm{CH})$ con propiedades físicas y mecánicas muy similares a las presentadas en este artículo. Las muestras recuperadas fueron sometidas a ensayos de columna resonante con diferentes valores de presión de confinamiento. En la tabla 8 se relacionan las características del sondeo M1, el cual reporta una profundidad de exploración cercana a $6,00 \mathrm{~m}$, acorde con los 2 sondeos realizados para este artículo, por lo cual es posible asumir un comportamiento dinámico igualmente similar.

Tabla 8 Sondeo M1, Fuente: [20]

\begin{tabular}{|c|c|c|c|c|l|c|}
\hline MUESTRA & PROFUNDIDAD & USCS & $\omega(\%)$ & $\mathrm{S}(\%)$ & DESCRIPCION & NOMENCLATURA \\
\hline \multirow{2}{*}{$\begin{array}{c}\text { ARCILLA } \\
\text { Color gris }\end{array}$} & \multirow{3}{*}{$5,85 \mathrm{~m}$} & \multirow{2}{*}{$\mathrm{CH}$} & 104,41 & 100,00 & HUMEDAD NATURAL & M1-1 \\
\cline { 4 - 7 } & & 95,56 & 96,91 & PTO ANTES DEL VEA & M1-2 \\
\cline { 5 - 7 } & & 67,25 & 80,53 & PTO DESPUES DEL VEA & M1-3 \\
\hline
\end{tabular}

A continuación, se muestra en la figura 31 la variación del módulo de corte máx. del suelo a diferentes valores de presión de confinamiento obtenidos mediante ensayos de columna resonante. 


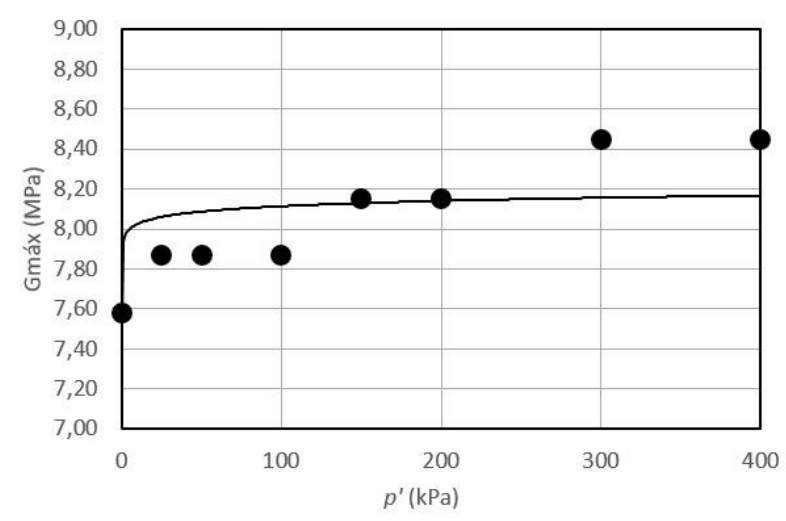

- Gmax -Potencial (Gmax)

Fig. 31 Variación del módulo de corte con respecto a la presión de confinamiento

Como consecuencia de la aplicación de diferentes valores torsionales bajo una presión de confinamiento de $400 \mathrm{KPa}$. Se observa la variación de las propiedades dinámicas del suelo en estado natural (muestra M1-1). en la tabla 9.

Tabla 9 Propiedades Dinámicas Muestra 1-1, Fuente: [20]

\begin{tabular}{|c|c|c|c|c|}
\hline \multicolumn{5}{|c|}{ Propiedades dinámicas muestra M1; $\boldsymbol{p}^{\prime}=\mathbf{4 0 0} \mathrm{kPa}$} \\
\hline \multicolumn{2}{|c|}{ Torque aplicado } & \multicolumn{3}{|c|}{ M1-1 } \\
\hline$t^{\prime}(p f s)$ & $t^{\prime}(\mathrm{N}, \mathrm{m})$ & Gmáx $(\mathrm{MPa})$ & $(\mathrm{G} / G m a ́ x)$ & $\gamma(\%)$ \\
\hline 1 & 0,023 & 8,45 & 1,00 & 0,061 \\
\hline 2 & 0,046 & 6,76 & 0,80 & 0,12 \\
\hline 3 & 0,069 & 5,03 & 0,60 & 0,18 \\
\hline 4 & 0,092 & 4,37 & 0,52 & 0,25 \\
\hline 5 & 0,115 & 3,75 & 0,44 & 0,32 \\
\hline 6 & 0,138 & 3,19 & 0,38 & 0,41 \\
\hline 7 & 0,161 & 2,66 & 0,31 & 0,51 \\
\hline
\end{tabular}

Teniendo en cuenta el objetivo propuesto: "analizar el efecto de fuerzas dinámicas aplicadas por equipos de construcción sobre suelos blandos de la sabana de Bogotá"; y con base en las curvas de resonancia del material (figura 32), se puede observar que cuando el suelo estudiado en su estado natural es sometido a ensayos de columna resonante bajo las condiciones de la tabla 9 , la frecuencia de resonancia del suelo oscila entre $15 \mathrm{~Hz}$ y $29 \mathrm{~Hz}$ aproximadamente.

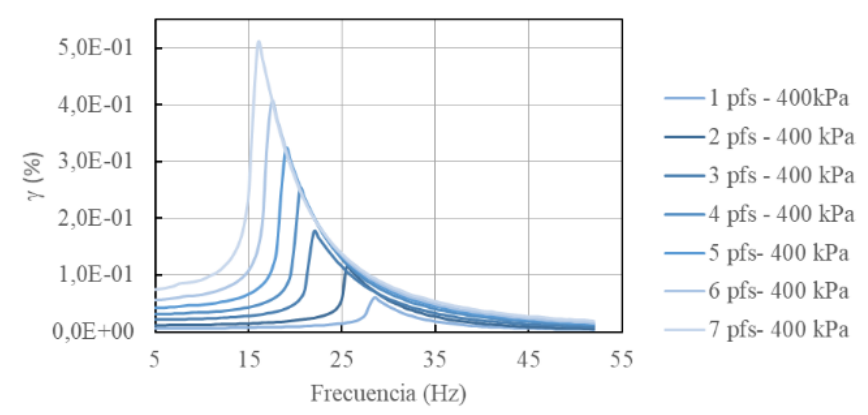

Fig. 32 Curvas de resonancia del suelo en estado natural, Fuente: [20]

\section{INFLUENCIA DE LAS VIBRACIONES EN LAS PROPIEDADES DEL PERFIL DE SUELO}

A partir de las propiedades dinámicas del suelo y sus frecuencias de resonancia relacionadas en la figura 32 es posible establecer su comportamiento dinámico al aplicarle cargas vibratorias ejercidas por equipos utilizados en construcción durante su tiempo de operación en contacto con el suelo. A continuación, se analiza el efecto producido por diferentes tipos de vibro-compactadores y martillo hidráulico el cual es utilizado en el hincado de pilotes mediante percusión.

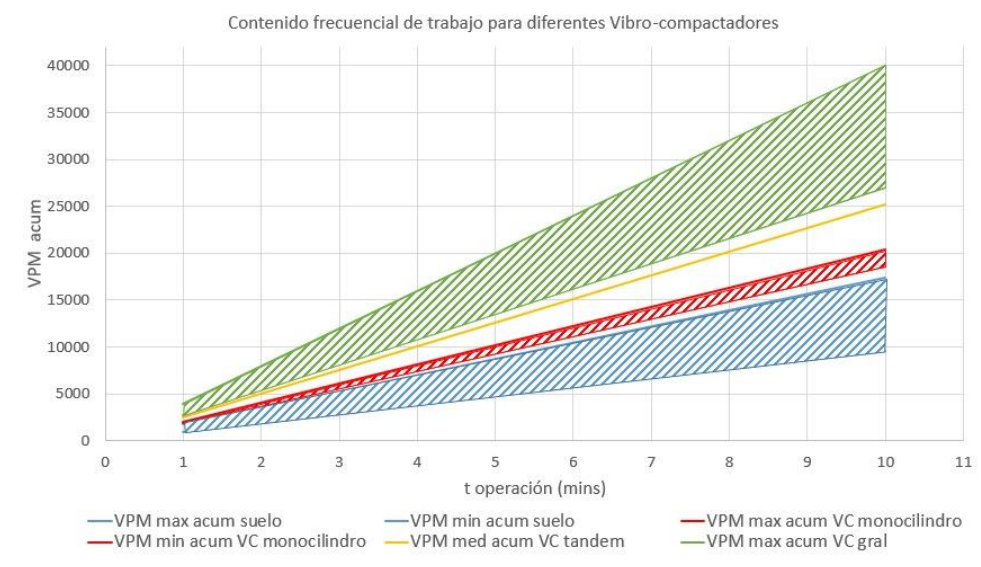

Fig. 33 Interacción de frecuencias vibratorias emitidas por Vibrocompactadores vs frecuencia de resonancia del suelo estudiado.

En la figura 33 se observa la interacción que ejerce el contenido frecuencial de diferentes tipos de vibro-compactadores con el suelo estudiado, en donde se observa que los vibro-compactadores monocilíndricos manejan un rango de vibraciones de trabajo muy cercano al resistido por el suelo, por el contrario, los vibro compactadores en tándem y aquellos que manejan múltiples frecuencias de trabajo parecen estar cada vez más lejos de afectar el suelo. La información frecuencial se obtuvo de algunos de los manuales de las maquinas, donde se obtuvo que para compactadores pata de cabra el rango va entre 31 y $34 \mathrm{~Hz}$, mientras que para compactadores tándem hay una media de $42 \mathrm{~Hz}$, finalmente para los compactadores mono-cilíndricos el rango esta entre $45 \mathrm{~Hz}$ y $67 \mathrm{~Hz}$. [21]

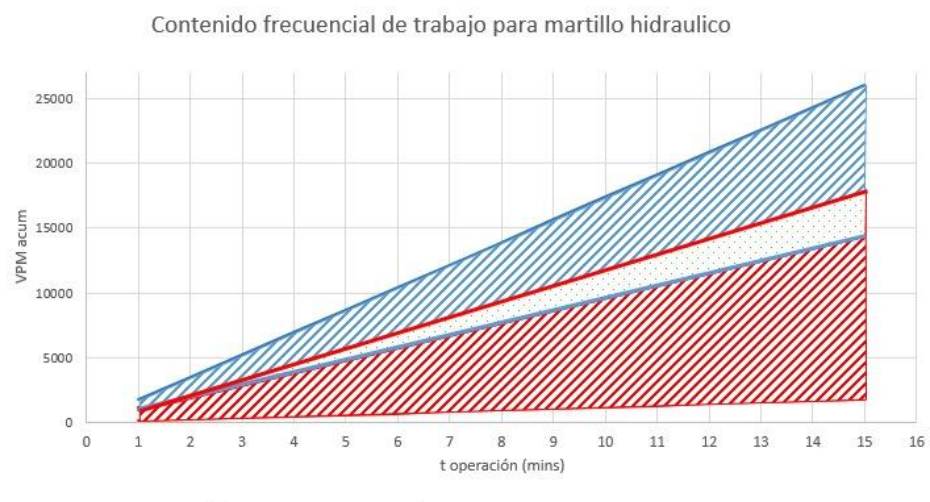

—VPM max acum suelo —VPM min acum suelo —VPM max acum maq _ -VPM min acum maq

Fig. 34 Interacción de frecuencias vibratorias emitidas por martillo hidráulico vs frecuencia de resonancia del suelo estudiado.

Contrario a la figura 33, de la figura 34 se infiere un comportamiento de resonancia a partir de la interacción de contenido frecuencial 
emitido por la percusión en cada golpe del martillo hidráulico vs la frecuencia de resonancia del suelo estudiado, teniendo en cuenta que la frecuencia máxima de trabajo de la maquina interfiere con la frecuencia mínima de resonancia del suelo, es posible asumir una potencial resonancia entre los elementos.

Con el fin de hacer un análisis de frecuencia vs deformación del suelo se tomaron unos valores pico de graficas de amplitud espectral vs frecuencia, [10], estos valores fueron varios puntos de frecuencias máximas que se observaron en distintos casos, como en hincado de pilotes, en trenes y tráfico pesado. Tal y como se observa en la figura 35 .
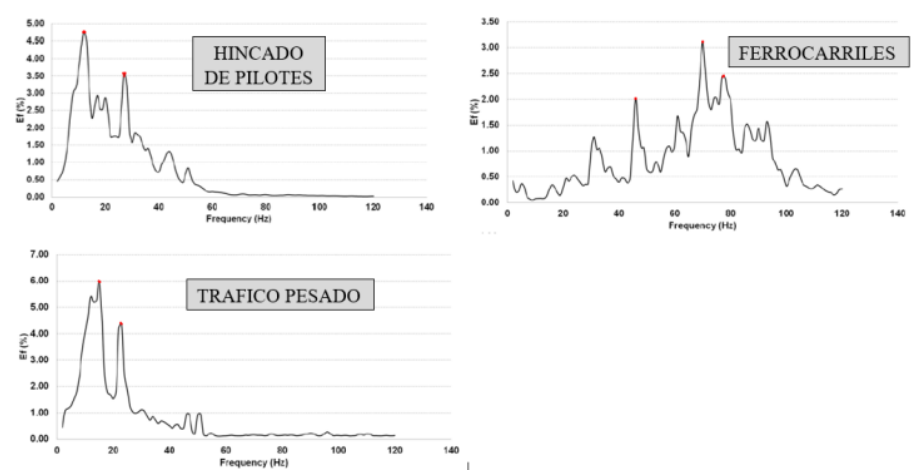

Fig. 35 Distribución relativa de la amplitud por frecuencia para Hincado de pilotes, trenes y tráfico pesado [10].

A través del factor R, el cual es hallado mediante la ecuación $\mathrm{N}^{\circ} 1$, es posible determinar el comportamiento dinámico del suelo para distintas obras civiles que involucran aspectos geotécnicos con base en las deformaciones que estas emiten sobre el suelo durante la ejecución de actividades propias de la ingeniería civil.

$$
R=\frac{f(h z) \text { suelo }}{f(h z) \text { fuente }}
$$

\section{Ecuación 1 Cálculo de factor $R$}

El valor crítico para el factor $\mathrm{R}$ es 1 , ya que este valor indica una potencial resonancia de los elementos al igualarse las frecuencias de trabajo de cada actividad con la frecuencia de resonancia del suelo.

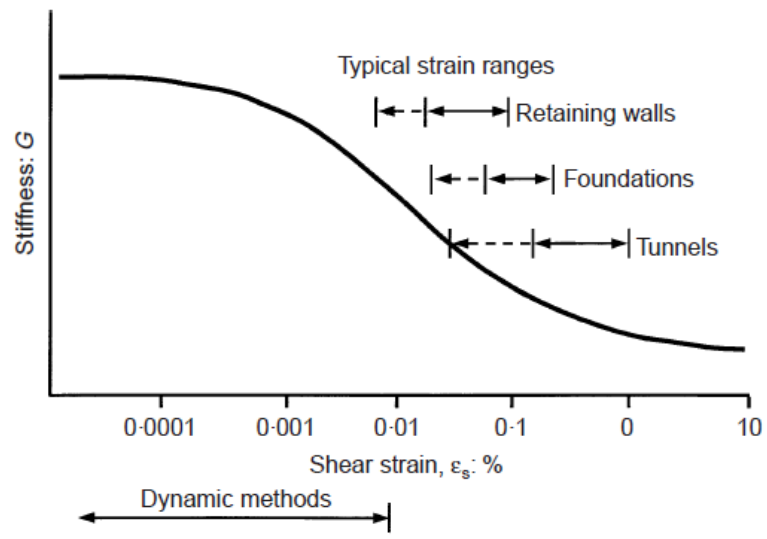

Fig. 36 Comportamiento característico rigidez \pm deformación del suelo con rangos de tensión típicos para pruebas de laboratorio y estructuras. [22]

Así mismo se tuvo en cuenta las deformaciones conocidas de algunas estructuras como se puede apreciar en la figura 36, esto con el fin de evaluar el comportamiento del suelo y las posibles afectaciones que se podrían generar por fuerzas dinámicas aplicadas, como las producidas por maquinaria pesada, tráfico vehicular, pesado y corredores férreos.

Tabla 10 Diferentes tipos de cargas dinámicas analizadas.

\begin{tabular}{|l|l|}
\hline VC & Vibrocompactadores \\
\hline PH & Pilotes hincados \\
\hline Vol & Voladura \\
\hline Tv & $\begin{array}{l}\text { Tráfico vehicular de } \\
\text { diseño (tráfico } \\
\text { pesado) }\end{array}$ \\
\hline Trenes & Ferrocarriles \\
\hline
\end{tabular}

En la tabla 10 se puede apreciar la nomenclatura que fue utilizada para analizar el comportamiento de la influencia de vibraciones (Factor $\mathrm{R}$ vs deformación, figura 37)

Influencia de vibraciones

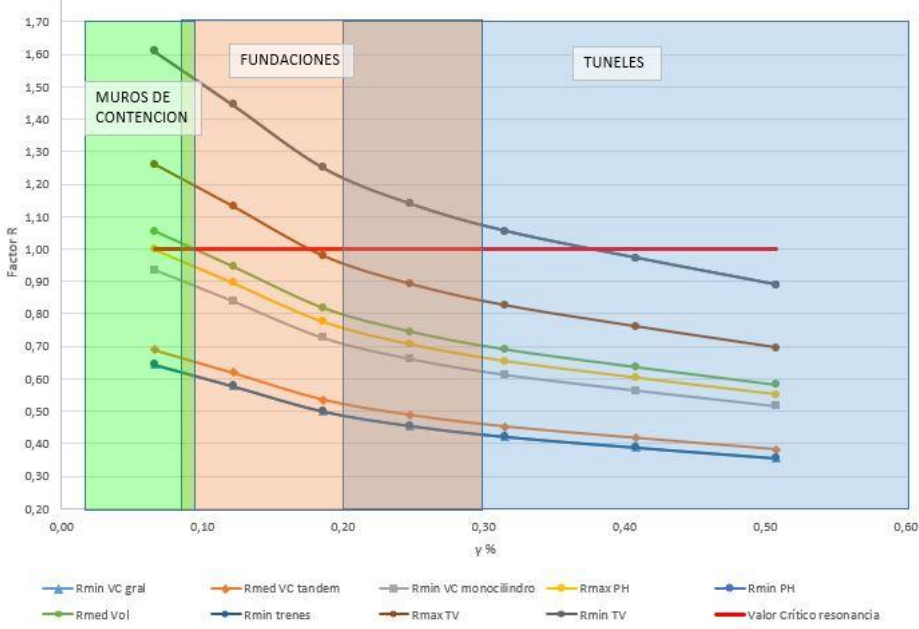

Fig. 37. Factor $R$ a diferentes valores de deformación angular para distintas actividades propias de la ingeniería civil.

Como resultado del análisis presentado en la figura 37, el cual exhibe la condición más crítica para cada actividad planteada, se determina que para la zona de estudio podrían presentarse problemas asociados a resonancia del suelo en actividades de pilotaje, voladura e incluso el mismo tráfico vehicular que por allí circula teniendo en cuenta que esta se encuentra en cercanías a una vía secundaria de orden departamental. Por otro lado, las actividades que incluyen el uso de vibrocompactadores reflejan un buen comportamiento del suelo al igual que el uso y la circulación de trenes por esta zona.

En las franjas de color se muestran los niveles de deformación que imponen sobre el suelo diferentes estructuras tales como muros de contención, fundaciones o túneles. Las cargas impuestas por estas estructuras son monotónicas, de manera que luego de que el suelo alcanza el nivel de deformación, se mueve por la superficie de cedencia acercándose a fallar, y como se vio, también aumenta la frecuencia de resonancia, de manera que el depósito en la vecindad de la estructura, cambia su respuesta ante cargas dinámicas. Es claro que para el nivel de deformaciones que imponen las fundaciones superficiales, también se observa una susceptibilidad a la convergencia entre las frecuencias de resonancia del suelo y la que imponen los vehículos y los trenes. Esto es particularmente importante, si se tiene en cuenta que esta zona de la sabana es conocida por presentar problemas de estabilidad en el tiempo de su malla vial; asociada hasta ahora a problemas relacionados con la desecación y cambios en el contenido de agua, pero es claro según los resultados que una posible implicación en las inestabilidades es el hecho de que las frecuencias de vibración de los vehículos pueden 
incidir en el reblandecimiento de los depósitos aumentando los asentamientos.

\section{CONCLUSIONES}

A partir de la necesidad de interpretar los efectos de fuerzas dinámicas aplicadas por equipos de construcción sobre el suelo estudiado, surge la implementación del gráfico de la Figura37, el cual muestra ser confiable para describir el comportamiento de un suelo de la sabana de Bogotá con un origen lacustre, frente a cargas dinámicas impuestas a partir de ensayos de columna resonante. Como resultado de esta investigación se concluye que el factor $\mathrm{R}$ puede ser utilizado en estudios futuros similares y/o en los estudios previos que se pretendan realizar para obras civiles en esta zona o en zonas con características geotécnicas, geológicas y sísmicas similares debido a que este presenta resultados que pueden ser útiles en la predicción de consecuencias asociadas a la construcción de diferentes obras civiles, teniendo en cuenta el rango de deformaciones típicas que las mismas provoquen sobre el suelo y el contenido frecuencial que emiten las actividades ejecutadas propias de cada tipo de obra y su respectiva maquinaria y equipos utilizados.

Es posible evidenciar que en este tipo de suelo arcilloso el hincado de pilotes mediante percusión afecta en todo su rango de trabajo el comportamiento dinámico del suelo llevándolo a una potencial resonancia, por lo tanto se puede concluir que la construcción de cimentaciones en esta zona y bajo este método puede afectar seriamente las construcciones aledañas que para este caso incluyen una vía secundaria de orden departamental la cual comunica a la ciudad capital de Bogotá con los municipios de la sabana occidente y edificaciones del sector industrial que por su naturaleza transmiten cargas altas sobre el suelo y podrían llegar a sufrir afectaciones que las desestabilicen.

En la gráfica de influencia de vibraciones obtenida en la figura $37 \mathrm{se}$ aprecia que la interacción de contenido frecuencial de trenes con el suelo presenta un comportamiento mucho mejor al generado por el tráfico vehicular, dicho concepto base puede conducir a la posibilidad de plantear a futuro la idea de un tren ligero que comunique a la ciudad de Bogotá con los municipios de la sabana occidente, en especial los aledaños a Tenjo.

Se concluye que el uso de vibrocompactadores posiblemente no incide en la generación de inestabilidades en este tipo de suelos $(\mathrm{CH})$, dado a que los rangos de trabajo no coinciden con el de las frecuencias que se encontraron en el suelo para el nivel de deformaciones. Lo que sí es evidente, es que en el rango de deformaciones que imponen las cimentaciones superficiales, es posible que se presente una coincidencia entre las frecuencias del suelo y las de los vehículos automotores, lo cual es particularmente interesante dado que en la zona estudiada son recurrentes los problemas viales relacionados con patologías asociadas a asentamientos que hasta la fecha se aducen a cambios en el contenido de agua, pero que según se vio, también pueden estar relacionados con el reblandecimiento dinámico del depósito.

Finalmente se demostró que hacer este tipo análisis y laboratorios dinámicos puede dar mejores ideas sobre el tipo de obras que se va a realizar en suelos arcillosos, por lo que se recomienda hacer énfasis en el análisis dinámico en cada estudio de suelos, esto con el fin de conocer a fondo todas las propiedades del suelo, y evaluar todos los posibles riesgos que se dan a causa de construcciones en ingeniería civil en el que se usa maquinaria pesada, lo que podría conllevar a una mayor eficiencia en las obras.

\section{REFERENCIAS}

[1] J. A. Diaz Rodríguez, Dinámica de suelos, Ciudad de Mexico: Limusa, 2005.

[2] C. A. Juárez Alvarado, «Estudio analítico de los efectos causados por el aisla de las vibraciones en cimentaciones para maquinaria industrial,» Mexico, 199

[3] J. Camacho-Tauta, «Comparison between exact and approximated viscoelastic transfer functions for a resonant-column system.,» Ingeniería $\mathrm{Y}$ Desarrollo, Bogotá, 2013

[4] D. A. Murcia Lopez y L. M. Perdomo Cruz, «Implementación del ensayo de columna resonante,» Universidad militar nueva granada, Bogotá, 2012.

[5] K. Ishihara y A. M. Ansal, «Dynamic behavior of soils, soil amplification and soil-structure interaction,» UNESCO, París, 1982.

[6] V. Kitovas, G. Stelmokaitis y V. Doroševas, «Investigation of Vibrations Influence on Clay Soil Parameters,» Journal of Sustainable Architecture and Civil Engineering, Kaunas, 2016.

[7] K. R. Massarsch, «Settlements and damage caused by construction-induced vibrations,» Worskshop wave , Alemania, 2000.

[8] JS HELD, «The Impact of Construction Vibration on Adjacent Structures,» JS HELD UNIVERSITY, Nueva York, 2019.

[9] H. Amick, M. ASCE y M. Gendreau, «Construction Vibrations and Their Impact on Vibration-Sensitive Facilities,» University of california, California, 2015.

[10] A. K. Jamal Eddine, «Sources of vibrations and their impact on the environnement,» Université Paris-Est, Paris, 2017.

[11] J. E. Alva Hurtado y R. Salinas Basualdo, Dinámica de suelos: Estudio comparativo de metodologías para el análisis de cimentación de maquinarias, Perú: Universidad nacional de ingeniería, 1997.

[12] J. Duque Ramírez, «Estimación del riesgo de daño debido a vibraciones,» Universidad del valle, Cali, 2013.

[13] Alcaldia Mayor De Bogotá, «La construcción en el área metropolitana de Bogotá,» Bogotá, 2015

[14] J. Gómez, N. E. Montes, Á. Nivia y \& Diederix, «Plancha 5-09 del Atlas Geológico de Colombia 2015. Escala 1:500 000. .,» Servicio Geológico Colombiano, Bogotá, 2015.

[15] Instituto Distrital de Gestión de Riesgos y Cambio Climático - IDIGER, «Zonificación de la respuesta sismica de Bogotá para el diseño sismo resistente de edificaciones,» Alcaldía Mayor de Bogotá, Bogotá, 2010.

[16] Ministerio de Ambiente, Vivienda y Desarrollo territorial, «Reglamento colombiano de construcción sismo resistente (NSR-10),» Ministerio de Ambiente, Vivienda y Desarrollo territorial, Bogotá, 2010.

[17] Alcaldia Mayor de Bogotá, «Decreto 523,» Alcaldia Mayor de Bogotá, Bogotá, 2010.

[18] Ingeominas y Universidad de los Andes, «Microzonificación sísmica de Santa Fe de Bogotá D. C.,» Ingeominas, Bogotá, 1997. 
[19] S. A. Rivera Pardo y L. L. Rojas Perilla, «Caracterización de los suelos arcillosos desecados al occidente de la Sabana de Bogotá,» Universidad Santo Tomás, Bogotá, 2014.

[20] P. Y. Ávila, J. A. Pineda y J. A. Cruz, «Dynamic Behavior of Naturally Desiccated Clays Via Resonant Column Testing at Constant Water Content,» Universidad Santo Tomás, Bogotá, 2015.

[21] CAT, «Caterpillar,» 2018. [En línea]. Available: https://www.cat.com/es_MX/products/new/equipment/compactors/vibratorysoil-compactors.html. [Último acceso: 18 Marzo 2019].

[22] J. H. Atkinson, «Non-linear soil stiffness in routine design.,» Géotechnique, USA, 2000.

[23] J. P. Bardet, «Experimental soil mechanics,» Prentice Hall, New Jersey, 1997.

[24] M. Budhu, «Soils Mechanics and foundations,» JOHN WILEY \& SONS, INC, Arizona, 2011.

[25] F. Amini, «Dynamic soil properties using improved transfer function methods,» University of the District of Columbia, USA, 1990.

[26] J. Camacho Tauta, «Evaluation of the small-strain stiffness of soil by nonconventional dynamic testing methods,» Lisboa, 2011.

[27] J. A. Diaz Rodriguez, «Course on seismic engineering and soil dynamic,» Universidad Nacional Autónoma De México, Ciudad de México, 2013.

[28] C. García, H. Monsalve, D. Peña y M. Pareja, «Ajuste de curvas de degradación de módulo de cortante y relación de amortiguamiento para los suelos de cenizas: caso Armenia (Colombia),» Revista de investigaciones Universidad del Quindío, Armenia, 2015.

[29] A. N. Guillermo y J. C. Santamarina, «Clasificación de suelos: Fundamento físico, prácticas actuales y recomendaciones,» Georgia Institute of Technology, Georgia, 2008

[30] S. L. Kramer, «Geotechnical Earthquake Engineering,» Prentice hall, Washington, 1996.

[31] S. L. Kramer, «Geotechnical Earthquake Engineering.,» Prentice Hall, Washington, 1996.

[32] G. Matos Montelli , «Matos Montelli , G. (2013). Análisis microestructural de la rigidez a pequeñas deformaciones de la bentonita febex en condiciones no saturadas,» Universidad politécnica de Cataluña, Barcelona, 2013.

[33] A. Verruijt, «Soil Dynamics.,» Delft University of Technology, Paises Bajos, 2008.

[34] J. R. Zetina Muñoz, «Diseño Práctico de cimentaciones sujetas a vibración producida por maquinaria,» Palibrio, New Jersey, 2013. 